\title{
Epigallocatechin-3-Gallate Alleviates High-Fat Diet-Induced Nonalcoholic Fatty Liver Disease via Inhibition of Apoptosis and Promotion of Autophagy through the ROS/MAPK Signaling Pathway
}

\author{
Dongdong Wu (iD, ${ }^{1,2}$ Zhengguo Liu, ${ }^{1}$ Yizhen Wang, ${ }^{1}$ Qianqian Zhang, ${ }^{1}$ Jianmei Li, \\ Peiyu Zhong, ${ }^{1}$ Zhongwen Xie $\mathbb{D}^{3},{ }^{3}$ Ailing Ji $\left(\mathbb{D},{ }^{1}\right.$ and Yanzhang $\mathrm{Li}(\mathbb{D})^{1}$ \\ ${ }^{1}$ Henan International Joint Laboratory for Nuclear Protein Regulation, School of Basic Medical Sciences, Henan University, Kaifeng, \\ Henan 475004, China \\ ${ }^{2}$ School of Stomatology, Henan University, Kaifeng, Henan 475004, China \\ ${ }^{3}$ State Key Laboratory of Tea Plant Biology and Utilization, Anhui Agricultural University, Hefei, Anhui 230036, China
}

Correspondence should be addressed to Zhongwen Xie; zhongwenxie@ahau.edu.cn, Ailing Ji; ailingji@163.com, and Yanzhang Li; yanzhang206@163.com

Received 21 January 2021; Revised 22 March 2021; Accepted 28 March 2021; Published 17 April 2021

Academic Editor: Stefania D’Adamo

Copyright (C) 2021 Dongdong Wu et al. This is an open access article distributed under the Creative Commons Attribution License, which permits unrestricted use, distribution, and reproduction in any medium, provided the original work is properly cited.

Nonalcoholic fatty liver disease (NAFLD) represents one of the most common chronic liver diseases in the world. It has been reported that epigallocatechin-3-gallate (EGCG) plays important biological and pharmacological roles in mammalian cells. Nevertheless, the mechanism underlying the beneficial effect of EGCG on the progression of NAFLD has not been fully elucidated. In the present study, the mechanisms of action of EGCG on the growth, apoptosis, and autophagy were examined using oleic acid- (OA-) treated liver cells and the high-fat diet- (HFD-) induced NAFLD mouse model. Administration of EGCG promoted the growth of OA-treated liver cells. EGCG could reduce mitochondrial-dependent apoptosis and increase autophagy possibly via the reactive oxygen species- (ROS-) mediated mitogen-activated protein kinase (MAPK) pathway in OAtreated liver cells. In line with in vitro findings, our in vivo study verified that treatment with EGCG attenuated HFD-induced NAFLD through reduction of apoptosis and promotion of autophagy. EGCG can alleviate HFD-induced NAFLD possibly by decreasing apoptosis and increasing autophagy via the ROS/MAPK pathway. EGCG may be a promising agent for the treatment of NAFLD.

\section{Introduction}

Tea, made from the leaves of Camellia sinensis, has long been considered a popular beverage worldwide [1-3]. Tea can be mainly classified into three types according to the manufacturing processes, including green tea (nonfermented), oolong tea (semifermented), and red and black teas (fermented) [4]. The functional constituents of tea can be attributable to the polyphenolic compounds, particularly catechins [1]. Four main catechins have been identified in green tea, such as epigallocatechin-3-gallate (EGCG), epigallocatechin, epicatechin-3-gallate, and epicatechin, with EGCG as the most active and abundant compound $[3,5]$. These catechins have different hydroxyl groups on the B-ring with the presence/absence of a galloyl moiety [4]. EGCG exhibits strong binding to bioactive macromolecules, such as DNA and proteins via $\pi-\pi$ stacking interaction, hydrogen bonding, and hydrophobic interaction $[5,6]$.

EGCG, a flavone-3-ol phenolic compound, has eight free hydroxyl groups [2], which might contribute to its diverse 
biological and pharmacological properties, such as antiamyloidogenic [7], chemopreventive [8], renoprotective [9], anticancer [10], antiaging [11], antiautoimmune [12], and antiviral [13] activities. Nonalcoholic fatty liver disease (NAFLD), a common chronic liver disease, has been considered one of the leading causes of end-stage liver disease, liver transplantation, and hepatocellular carcinoma [14-16]. The prevalence of NAFLD is growing in parallel with the global obesity epidemics, hypertension, type 2 diabetes, hyperlipidemia, and metabolic syndromes $[15,16]$. It has been shown that EGCG could attenuate high-fat diet- (HFD-) induced NAFLD in rats and mice [17-19]. Nevertheless, the inhibitory effects and detailed mechanisms of EGCG in the initiation and progression of NAFLD need to be further investigated.

In this study, the mechanism of action of EGCG on the growth, apoptosis, and autophagy of oleic acid- (OA-) treated liver cells was elucidated. The HFD-induced NAFLD mouse model was further adopted to confirm the effect and mechanism of EGCG on NAFLD.

\section{Materials and Methods}

2.1. Cell Culture. Human liver cell lines L02 and QSG-7701 were purchased from Feiya Biological Technology Co., Ltd. (Yancheng, Jiangsu, China). The cells were cultured in Dulbecco's modified Eagle's medium (DMEM) with 10\% fetal bovine serum (FBS) and 1\% streptomycin/penicillin. Before each experiment, the cells were starved for $12 \mathrm{~h}$ in serumfree DMEM. Then, the cells were treated with the medium containing the $0.5 \mathrm{mM}$ OA-bovine serum albumin (BSA; fatty acid-free, low endotoxin) complex (4:1, molar ratio), with or without a concentration of $50 \mu \mathrm{M}$ EGCG for $24 \mathrm{~h}$. The medium with only BSA was selected as the control [20].

2.2. Oil Red $O(O R O)$ Staining. Cells were fixed in $4 \%$ paraformaldehyde for $15 \mathrm{~min}$, incubated with isopropyl alcohol for $20 \mathrm{~min}$, and stained with ORO solution for $20 \mathrm{~min}$, followed by being counterstained with hematoxylin at room temperature. The staining intensity of ORO was measured by Image software (National Institutes of Health, Bethesda, MD, USA) [21].

2.3. Cell Growth Assay. The 5-ethynyl-2' -deoxyuridine (EdU) experiment was carried out using economical kits (RiboBio, Guangzhou, China). The cell proliferation rate was calculated as the percentage of positive cells to total cells. In addition, the cell counting kit-8 (CCK-8) detection kits (Beyotime, Shanghai, China) were used to detect cell viability. Cell viability was expressed as a percentage to the control group [22].

2.4. Flow Cytometry Assay. Cells were incubated with propidium iodide (PI)/RNase A mixture for $20 \mathrm{~min}$. A FACSVerse flow cytometer (BD, San Jose, CA, USA) was adopted to analyze the cell cycle. The apoptotic level was examined by Annexin VFITC/PI assay kits (KeyGen, Nanjing, Jiangsu, China) and further analyzed using a FACSVerse flow cytometer.

2.5. Immunofluorescence Staining. The green fluorescent protein- (GFP-)-red fluorescent protein- (RFP-) microtubule- associated protein 1 light chain 3 (MAP1LC3/LC3) plasmid has been used to detect the autophagic level [23]. Then, the GFP-RFP-LC3 plasmid (Hanbio, Shanghai, China) was transfected into the cells. After $48 \mathrm{~h}$ of incubation, the cellular fluorescence was determined using an Eclipse Ti fluorescent microscope (Nikon, Melville, NY, USA). The autophagosomes (yellow dots) and autolysosomes (red dots) were calculated as the ratios of positive-stained cells to total cells [24].

2.6. Monodansylcadaverine (MDC) Staining. Morphologically, the formation of autophagic vacuoles in the cytoplasm is a typical characteristic of autophagy. MDC is a key marker for autophagic vacuoles [25]. Briefly, the liver cells were stained with $50 \mu \mathrm{mol} / \mathrm{L} \mathrm{MDC} \mathrm{for} 30 \mathrm{~min}$ at $37^{\circ} \mathrm{C}$. Then, the cells were fixed with $5 \%$ paraformaldehyde and immediately observed under an Eclipse Ti fluorescent microscope (Nikon).

2.7. Measurement of Reactive Oxygen Species (ROS). Cellular ROS levels were measured by $2^{\prime}, 7^{\prime}$-dichlorodihydrofluorescein diacetate (Beyotime).

2.8. Determination of Antioxidant Activity. The total superoxide dismutase (SOD) activity was determined using the kit with WST-8 (Beyotime). The catalase (CAT) activity was analyzed using the CAT assay kit (Beyotime). The glutathione peroxidase (GSH-Px) activity was detected by the GSH-Px assay kit with nicotinamide adenine dinucleotide phosphate (Beyotime).

2.9. Western Blot. Western blot assay was adopted to determine the expression levels of proteins. The primary antibodies, such as anti-cyclin D1/E1, anti-cyclin-dependent kinase (CDK) 2/4, anti-p21, anti-p27, anti-beclin-1, anti-P62, anti-LC3A/B, antiextracellular signal-regulated protein kinase $1 / 2$ (ERK1/2), anti-phospho- (p-) ERK1/2 (Thr202/Tyr204), anti-c-Jun Nterminal kinase (JNK), anti-p-JNK (Thr183/Tyr185), antip38, and anti-p-p38 (Thr180/Tyr182), and the horseradish peroxidase-conjugated secondary antibody were purchased from Cell Signaling Technology (CST, Danvers, MA, USA). Anti-B-cell lymphoma-2 (Bcl-2), anti-Bcl-2-associated $\mathrm{X}$ protein (Bax), anti-B-cell lymphoma-extra large (Bcl-xl), anti$\mathrm{Bcl}$-xl/Bcl-2-associated death promoter (Bad), anti-cleaved caspase-3/9, anti-cleaved poly-ADP-ribose polymerase (PARP), and anti- $\beta$-actin were obtained from Proteintech (Chicago, IL, USA). The bands were detected with a chemiluminescence system (Thermo, Rockford, IL, USA). Band intensities were analyzed by densitometry using ImageJ software.

2.10. Animals. The animal experiment was approved by the Committee of Medical Ethics and Welfare for Experimental Animals of Henan University School of Medicine (HUSOM2017-208). C57BL/6J mice (8 weeks old, male), HFD (60\% $\mathrm{kcal}$ as fat), and low-fat diet (LFD, $10 \% \mathrm{kcal}$ as fat) were obtained from Vital River Laboratory Animal Technology Co., Ltd. (Beijing, China). All mice were maintained on a $12 \mathrm{~h}$ light/dark cycle and allowed access to food and water ad libitum. Mice were fed either HFD $(n=12)$ or LFD $(n=6)$ for a total of 14 weeks. After feeding for 10 weeks, HFD-fed mice were assigned to the HFD group $(n=6)$ and HFD + EGCG $(50 \mathrm{mg} / \mathrm{kg} / \mathrm{day})$ group $(n=6)$. The mice were treated 
for an additional 4 weeks. Food/water intakes and body weights of the mice were measured. Then, the mice were killed and blood samples were collected. The liver, brown fat, and white fat were removed and weighed.

2.11. Biochemical Analysis. The concentrations of triglyceride (TG), total cholesterol (TC), alanine aminotransferase (ALT), and aspartate aminotransferase (AST) in the plasma were examined by an automated hematology analyzer (BC6900, Mindray, Shenzhen, Guangdong, China). The contents of TG and TC in liver cells and tissues, as well as nonesterified fatty acid (NEFA) in liver tissues were detected by commercial enzyme-linked immunosorbent assay kits (Jiancheng Bioengineering Institute, Nanjing, Jiangsu, China).

2.12. Hematoxylin and Eosin (HE) Staining. Liver tissues were fixed in $10 \%$ neutral formalin, embedded in paraffin wax, sectioned at $4 \mu \mathrm{m}$, and then stained with HE.

2.13. Immunohistochemistry (IHC). Liver samples were respectively stained with anti-Ki67 (CST), anti-beclin-1, and anti-cleaved caspase- 3 antibodies. The proliferation index, autophagic index, and apoptotic index were determined by the ratios of positive cells to total cells.

2.14. Statistics. All data were presented as the mean \pm standard error of the mean. Differences between the two groups were determined by the two-tailed Student's $t$-test and one-way analysis of variance using GraphPad Prism 6 software. $P<0.05$ was considered to indicate a statistically significant difference.

\section{Results}

3.1. EGCG Promotes the Growth of OA-Treated Liver Cells. As shown in Figures 1(a) and 1(b), OA induced the accumulation of lipid in OA-treated liver cells, as further evidenced by the increased levels of TC and TG (Figures 1(c) and 1(d)). Treatment with EGCG reduced the lipid level in OAtreated liver cells (Figures 1(a)-1(d)). The viability and proliferation of liver cells were decreased by OA; nevertheless, EGCG promoted the viability and proliferation of OAtreated liver cells (Figures $1(\mathrm{e})-1(\mathrm{~g})$ ). In addition, the results showed that OA triggered cell cycle arrest at the G1 phase and EGCG reversed the trend (Figures 2(a) and 2(b)). Many cell cycle-related proteins have been identified in mammals, such as cell cycle regulatory proteins, including cyclin $\mathrm{D} 1 / \mathrm{E} 1$ and $\mathrm{CDK} 2 / 4$, as well as inhibitory cell cycle regulators, including p21 and p27 $[26,27]$. Our data suggested that OA increased the expressions of cyclin D1, cyclin E1, CDK2, and CDK4 but downregulated the protein levels of p21 and p27; however, administration of EGCG exhibited reverse trends (Figures 2(c) and 2(d)). Taken together, the data indicate that EGCG can promote the growth of OA-treated liver cells through promoting $\mathrm{G} 1$ phase cell cycle progression.

3.2. EGCG Decreases Apoptosis in OA-Treated Liver Cells. As shown in Figures 3(a) and 3(b), the data suggested that OA increased the early and late apoptotic cell populations, whereas EGCG decreased the early and late apoptosis in
OA-treated liver cells. The ratios of Bax/Bcl-2 and Bad/Bcl$\mathrm{xl}$ are regarded as key factors in regulating apoptosis. Increased ratios of $\mathrm{Bax} / \mathrm{Bcl}-2$ and $\mathrm{Bad} / \mathrm{Bcl}-\mathrm{xl}$ are key phenomena in mitochondrial-dependent apoptosis in mammals $[28,29]$. Furthermore, cleaved caspase-3/9 could induce apoptosis through the mitochondrial-mediated pathway [30]. PARP, a nuclear enzyme involved in DNA repair, is an important target for caspases during apoptosis [31]. The data showed that $\mathrm{OA}$ increased both the $\mathrm{Bax} / \mathrm{Bcl}-2$ and $\mathrm{Bad} / \mathrm{Bcl}-\mathrm{xl}$ ratios and the expression levels of cleaved caspase-3/9 and cleaved PARP, which were reversed by the administration of EGCG (Figures 3(c) and 3(d)). The results suggest that OA can induce mitochondrial-dependent apoptosis in liver cells and EGCG could reduce the apoptotic levels in OA-treated liver cells.

3.3. EGCG Increases Autophagy in OA-Treated Liver Cells. Autophagy is responsible for the degradation of intracellular protein aggregates, invasive pathogens, and damaged organelles and therefore is essential in maintaining cellular homeostasis and responding to stress conditions $[32,33]$. A crucial step in autophagy is the conversion of LC3 from the nonlipidated form (LC3-I) to the lipid-conjugated form (LC3-II) $[33,34]$. Autophagic turnover could be molecularly monitored using a GFP-conjugated LC3 and the conversion of LC3-I to LC3-II [35]. In the present study, the GFP-RFP-LC3 plasmid was transfected into liver cells and further detected by fluorescence microscopy. Treatment with OA decreased the numbers of free red dots (autolysosomes) and yellow dots (autophagosomes), whereas administration of EGCG showed the opposite effects (Figures 4(a) and 4(b)). A similar trend was observed in MDC staining (Figures 4(c) and 4(d)). Apart from LC3, beclin-1 and P62 have also been considered specific markers of autophagy [33, 36]. The expression levels of beclin-1 and LC3 in the OA group were lower than those in the control group, but the protein levels of these two factors were higher in the OA+EGCG group than in the OA group. Furthermore, the expression level of P62 exhibited a reverse trend (Figures 4(e) and 4(f)). These results together suggest that the autophagic level is downregulated in OA-treated liver cells and treatment with EGCG could upregulate the autophagy machinery.

\subsection{EGCG Suppresses the ROS/Mitogen-Activated Protein} Kinase (MAPK) Pathway in OA-Treated Liver Cells. ROS such as hydroxyl radical, hydrogen peroxide, and superoxide anion are normally generated as by-products of aerobic metabolism [37, 38]. ROS can be scavenged by the antioxidant defense system that mainly includes GSH-Px, SOD, and CAT [37-39]. Compared with the control group, the ROS levels were increased, but GSH-Px, SOD, and CAT activities were downregulated in the OA group, which can be reversed by the administration of EGCG (Figures 5(a) and 5(b)). The results suggest that EGCG can reduce OAinduced oxidative stress in liver cells. It has been shown that ROS can activate the MAPK pathway and attenuation of ROS by ROS scavengers could deactivate MAPK signaling [40, 41]. As shown in Figures 5(c) and 5(d), OA reduced the 


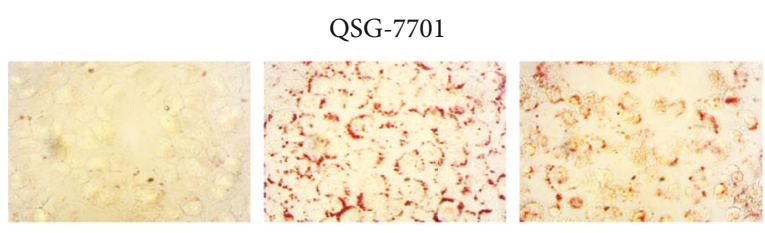

L02

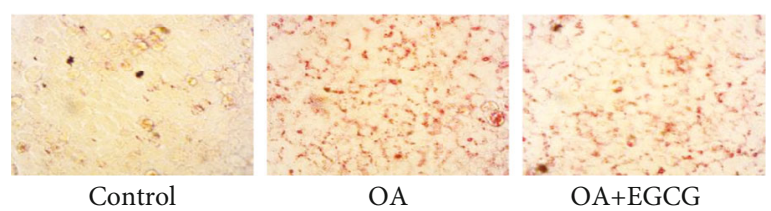

(a)
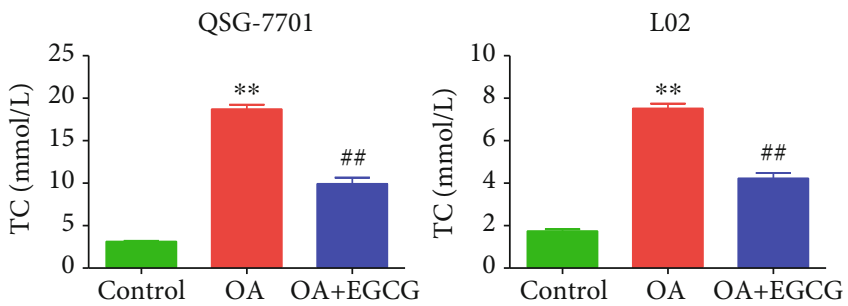

(c)

QSG-7701
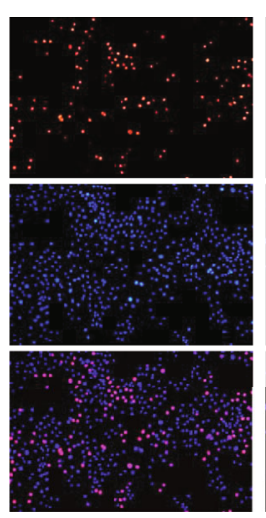

Control
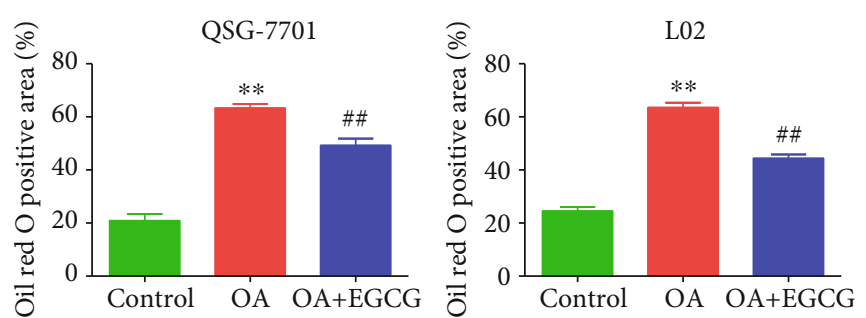

(b)
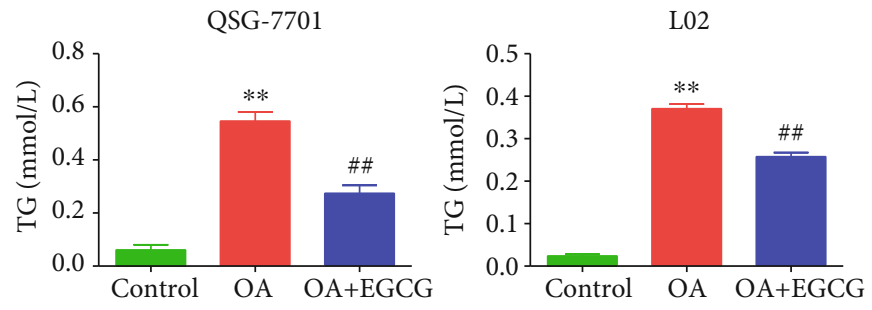

(d)
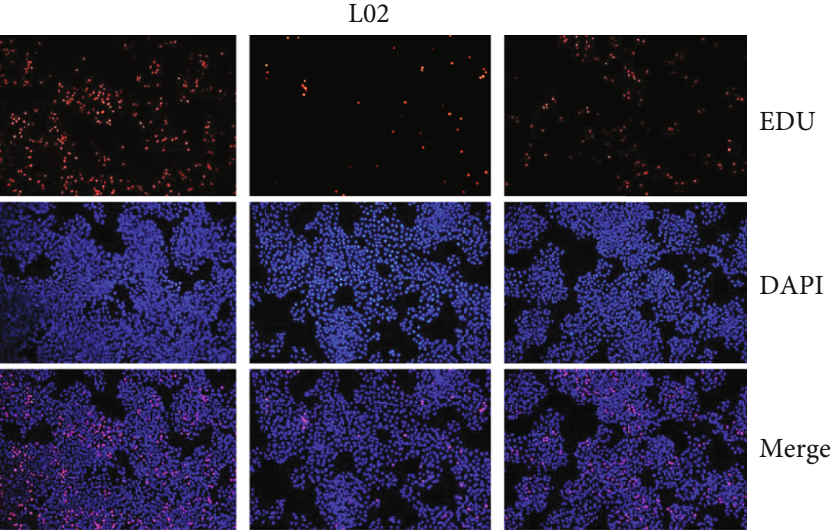

$\mathrm{OA}+\mathrm{EGCG}$

(e)
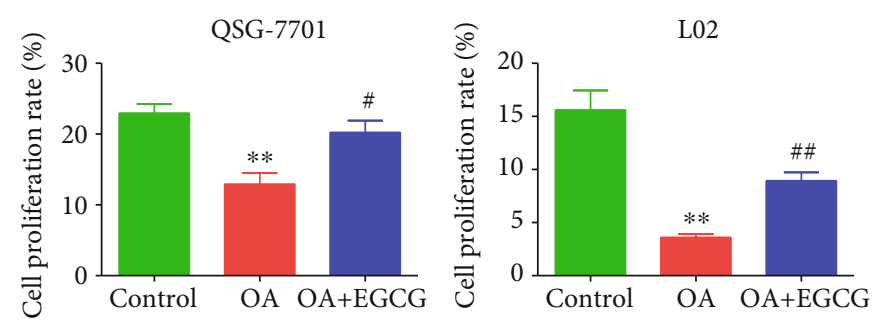

(f)

Figure 1: Continued. 


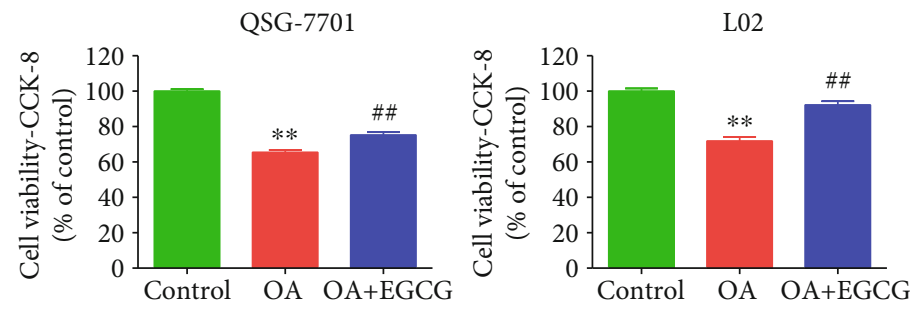

(g)

FIGURE 1: Effects of EGCG on lipid droplet formation and the growth of OA-treated QSG-7701 and L02 cells. (a) Representative photographs of ORO-stained QSG-7701 and L02 cells; original magnification $\times 400$. (b) ORO-positive area was calculated. (c) The levels of TC were measured. (d) The levels of TG were measured. (e) DNA replication activities were examined by EdU assay; original magnification $\times 200$. (f) The proliferation rate of each group was analyzed. (g) The percentages of viable cells were determined using CCK- 8 assay, and the cell viability of control cells was normalized as $100 \%$. Data are presented as mean \pm SEM of three independent experiments; ${ }^{* *} P<0.01$ compared with the control group; ${ }^{\#} P<0.05,{ }^{\# \#} P<0.01$ compared with the OA group.

expression of $\mathrm{p}$-ERK1/2 but increased the levels of $\mathrm{p}$-JNK and p-p38, while administration of EGCG exhibited reverse effects on the kinases. The data suggest that EGCG may suppress the ROS/MAPK pathway in OA-treated liver cells.

3.5. EGCG Attenuates HFD-Induced NAFLD in Mice. Compared with the mice fed with LFD for 10 weeks, HFD-fed mice exhibited increased body weight, indicating that an alimentary obesity model had been successfully established. In addition, compared to the LFD group, HFD-fed mice showed decreased food and water intakes, as well as increased weights of liver, white fat, and brown fat. Treatment with EGCG reversed these changes except for the food intake (Figures 6(a)-6(h)). Furthermore, HFD-fed mice exhibited increased levels of TC, TG, ALT, and AST when compared to the LFD group, which could be reversed by the treatment with EGCG (Figures 6(i)-6(l)). Moreover, HFD-fed mice showed upregulated levels of TC, TG, and NEFA in the liver when compared with the LFD group, which were reduced by the administration of EGCG (Figures 6(m)-6(o)). Compared to the LFD group, the HFD group exhibited a higher apoptotic index, as well as a lower proliferation index and autophagic index, which could be reversed by the administration of EGCG (Figures 7(a)-7(d)). These results indicate that EGCG can attenuate HFD-induced NAFLD in mice.

\section{Discussion}

NAFLD, the most common chronic liver disease, leads to end-stage liver disease, liver transplantation, and hepatocellular carcinoma [14-16]. It has been shown that EGCG plays important biological and pharmacological roles in mammals. Nevertheless, the effect and mechanism of EGCG in the process of NAFLD are largely unknown. Human normal liver cells QSG-7701 and L02 have been widely adopted to investigate the mechanism of action of novel drugs and donors [42, 43]. OA, a monounsaturated fatty acid, has been successfully used in the establishment of the NAFLD model [44]. In this study, QSG-7701 and L02 cells were adopted to examine the effects of EGCG on NAFLD induced by OA in vitro. A recent study has revealed that epoxy stearic acid, a type of oxidative product from $\mathrm{OA}$, can induce cytotoxicity and G0/G1 phase cell cycle arrest in HepG2 cells [45]. Our data indicated that $\mathrm{OA}$ decreased the viability and proliferation of liver cells and induced G1 phase cell cycle arrest. The changes could be reversed by the administration of EGCG. These data together indicate that EGCG acts as an effector molecule in promoting the growth of OA-treated liver cells.

Apoptosis is a conserved cell death pathway which can play key roles in the maintenance of organismal homeostasis and normal eukaryotic development [46, 47]. Two main apoptotic pathways have been identified in mammals: the mitochondrial-mediated intrinsic pathway and the death receptor-mediated extrinsic pathway [48]. Bcl-2 family proteins are involved in the regulation of apoptosis, such as Bax, Bad, Bcl-2, and Bcl-xl [49]. Many apoptotic stimuli can activate caspases, and PARP is activated by cleaved caspase-3, leading to the occurrence of apoptosis [31, 38, 49]. It has been reported that OA could induce apoptosis by increasing the levels of Bax and PARP but decreasing the level of Bcl-2 in HepG2 cells [50]. Another study shows that OA can promote the expressions of both cleaved caspase- 3 and PARP1 [51]. Similarly, our data indicated that OA induced the early and late apoptosis, as well as increased the ratios of both $\mathrm{Bax} / \mathrm{Bcl}-2$ and $\mathrm{Bad} / \mathrm{Bcl}-\mathrm{xl}$ and the expressions of cleaved caspase-3/9 and cleaved PARP in liver cells. EGCG significantly reduced the apoptotic levels in the OA group. The data suggest that the apoptotic levels are increased in OA-treated liver cells and treatment with EGCG could reduce apoptosis.

Autophagy, an evolutionarily conserved catabolic pathway, serves to deliver cytoplasmic materials to lysosomes for recycling and degradation, leading to macromolecular synthesis and energy production $[36,52]$. Autophagy is activated by many environmental factors, including cytokines, hormones, and nutrients [53]. Recent studies have indicated that autophagy is impaired in lipid-overloaded hepatocytes and in the liver from the NAFLD murine model and NAFLD patients [54-56]. In line with the above studies, we observed that the autophagic levels were decreased in OA-treated liver cells. Another study has reported that EGCG can increase the autophagic level by increasing lysosomal acidification and 


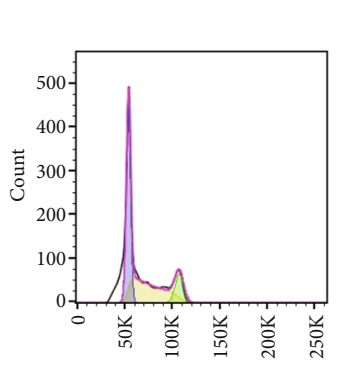

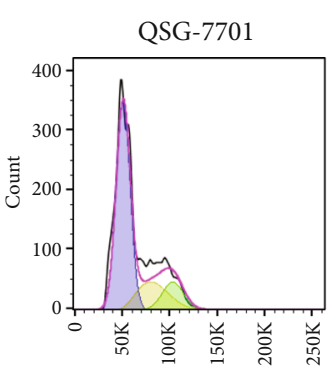

$\mathrm{OA}$

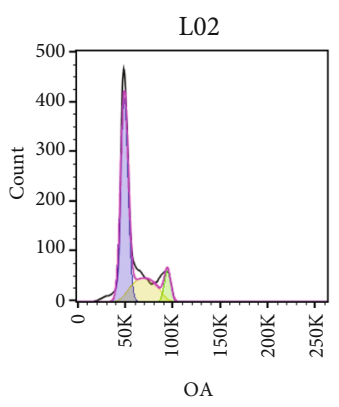

(a)
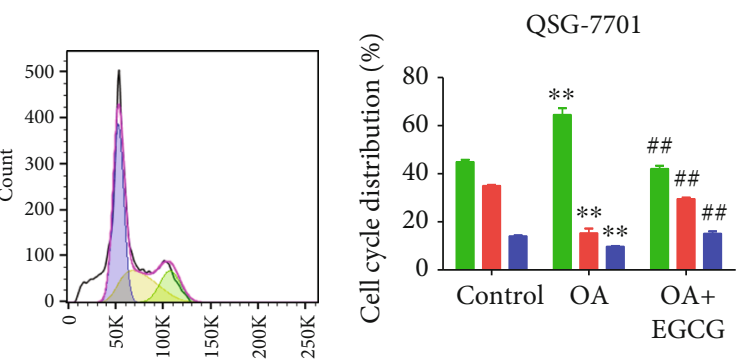

$\mathrm{OA}+\mathrm{EGCG}$
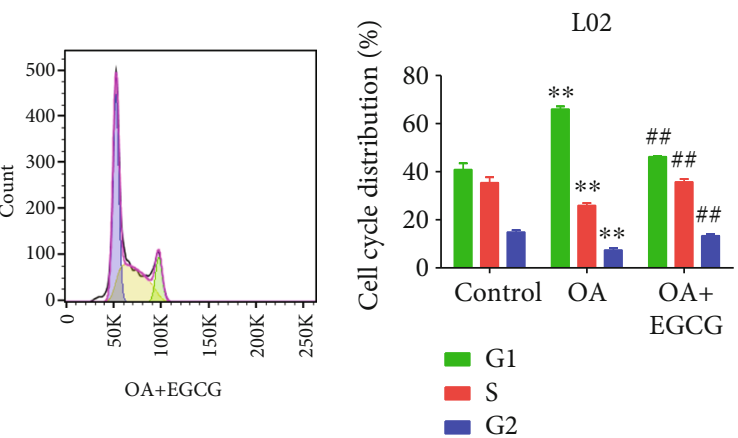

(b)
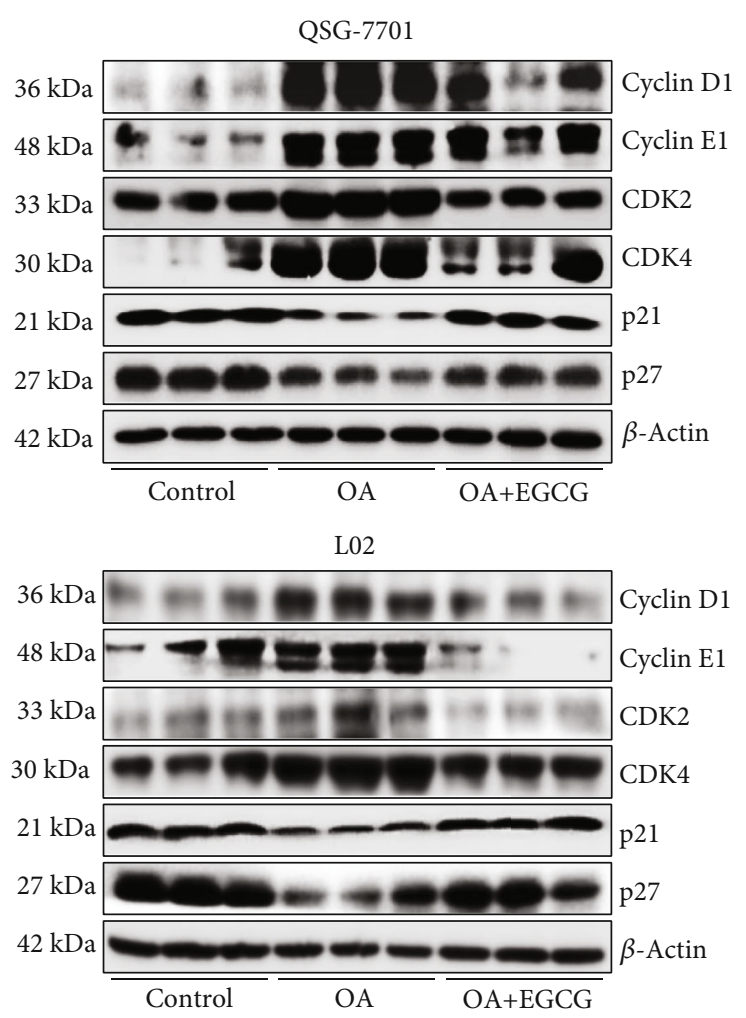

(c)
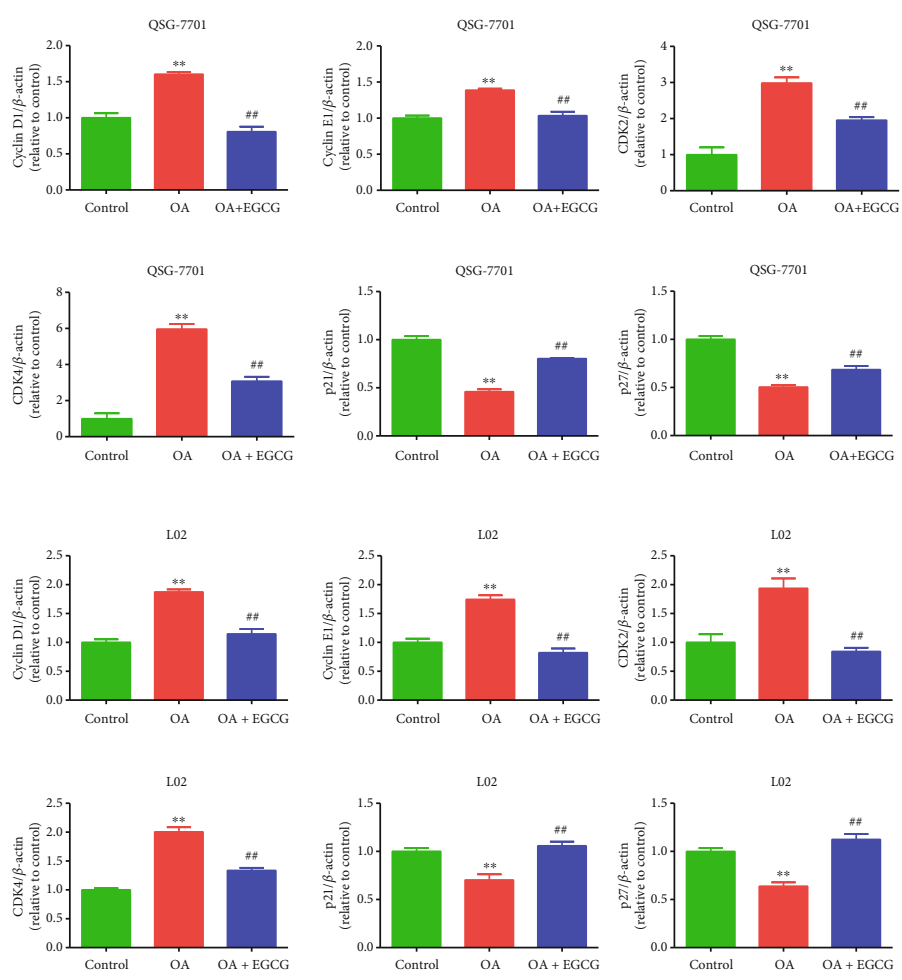

(d)

FIGURE 2: Effects of EGCG on cell cycle progression of OA-treated QSG-7701 and L02 cells. (a) Flow cytometry assay was used to determine cell cycle distribution. (b) Cell cycle distribution was analyzed. (c) Western blot analysis for the expression levels of cyclin D1, cyclin E1, CDK2, CDK4, p21, and p27 in each group. $\beta$-Actin was used as the loading control. (d) The densitometry analysis of each factor was performed in each group, normalized to the corresponding $\beta$-actin level. Data are presented as mean \pm SEM of three independent experiments; ${ }^{* *} P<0.01$ compared with the control group; ${ }^{\# \#} P<0.01$ compared with the OA group. 

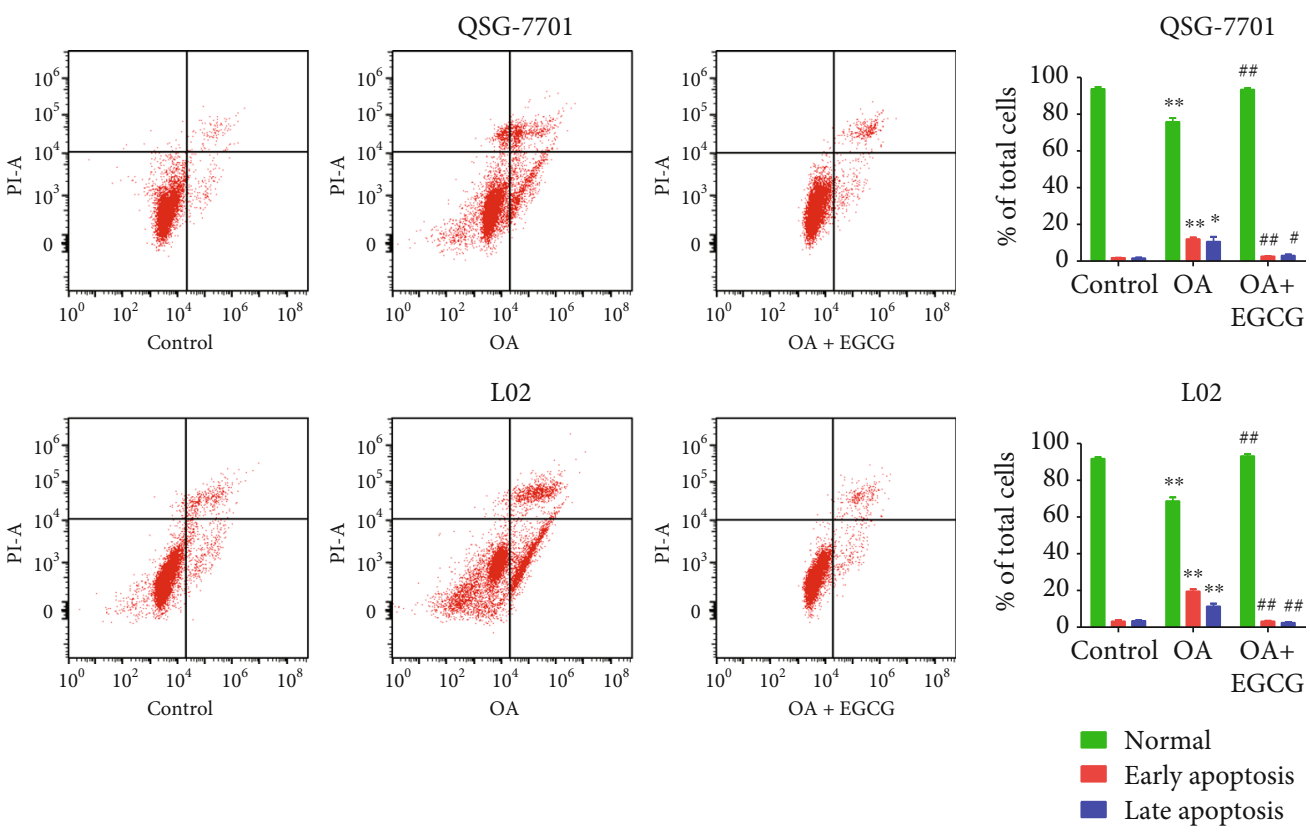

(a)

(b)
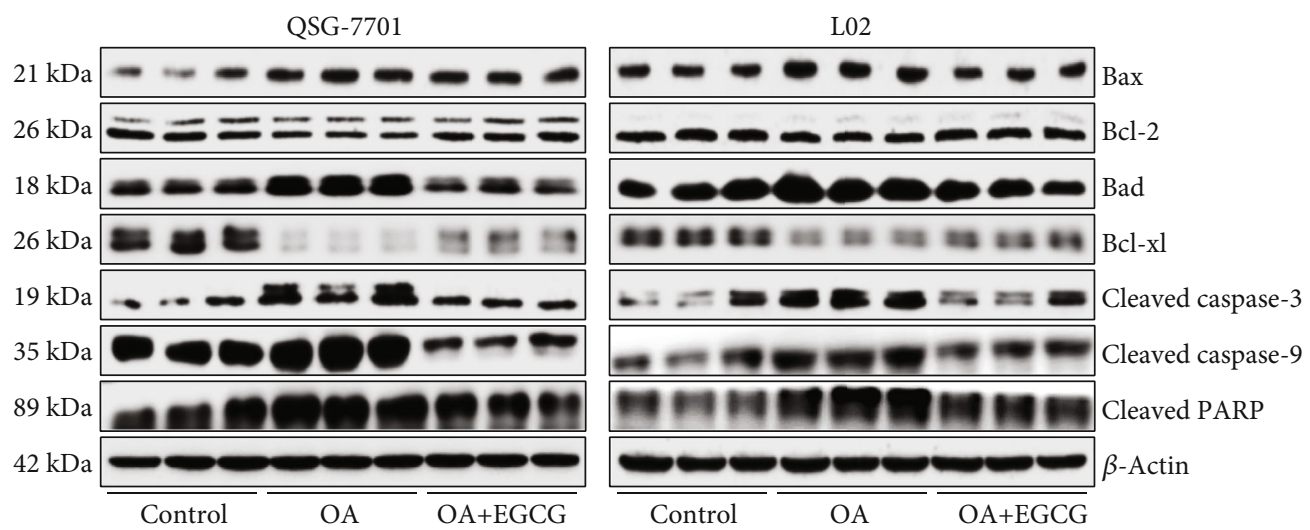

(c)
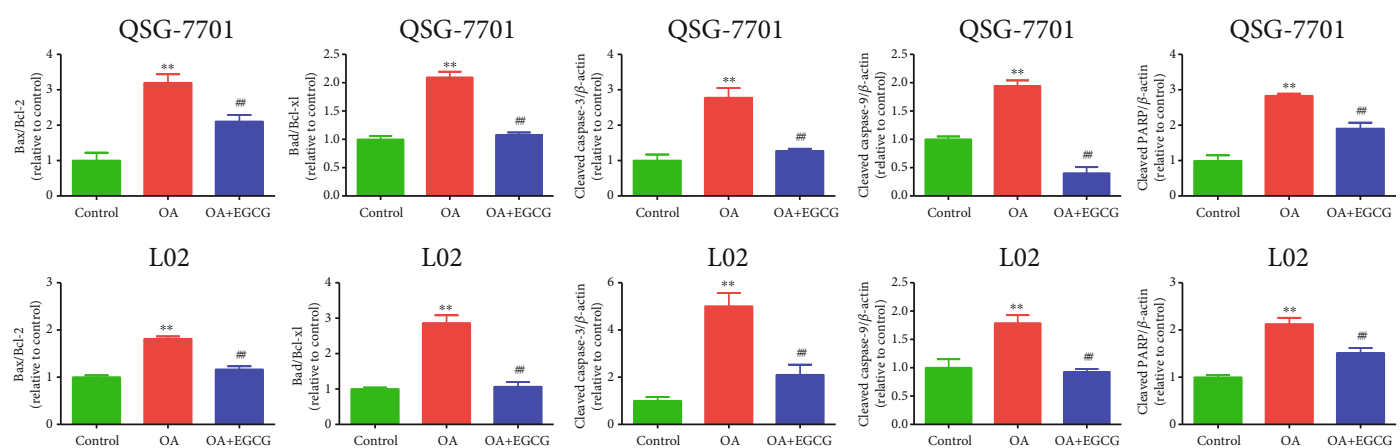

(d)

FIGURE 3: Effects of EGCG on the apoptosis of OA-treated QSG-7701 and L02 cells. (a) Flow cytometry assay was used to determine the apoptotic level. (b) The results of flow cytometry were analyzed. (c) Western blot analysis for the expression levels of Bax, Bcl-2, Bad, Bcl$\mathrm{xl}$, cleaved caspase-3/9, and cleaved PARP in each group. $\beta$-Actin was used as the loading control. (d) The densitometry analysis of each factor was performed in each group, normalized to the corresponding $\beta$-actin level. Data are presented as mean \pm SEM of three independent experiments; ${ }^{*} P<0.05,{ }^{* *} P<0.01$ compared with the control group; ${ }^{\#} P<0.05,{ }^{\# \#} P<0.01$ compared with the OA group. 
QSG-7701
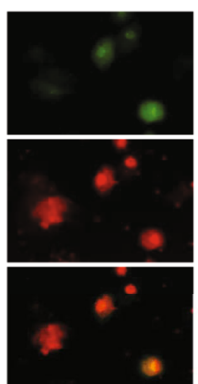

Control
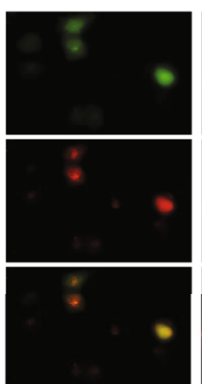

$\mathrm{OA}$
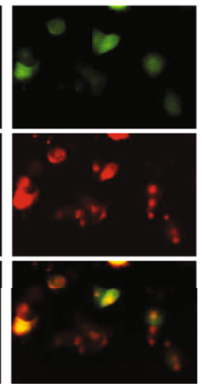

$\mathrm{OA}+\mathrm{EGCG}$
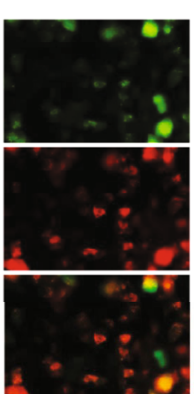

Control

L02

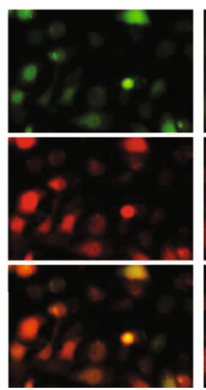

$\mathrm{OA}$

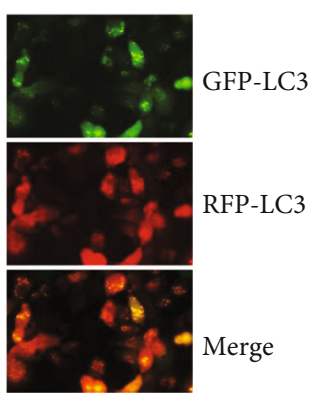

$\mathrm{OA}+\mathrm{EGCG}$

(a)

QSG-7701

QSG-7701
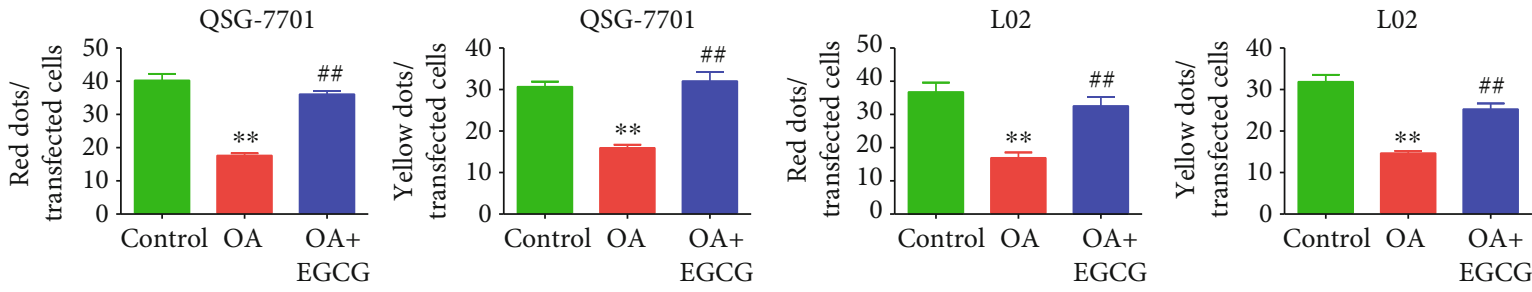

(b)

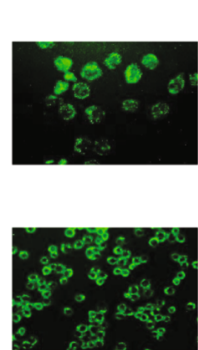

Control
QSG-7701

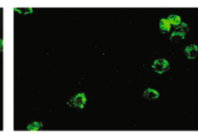

L02

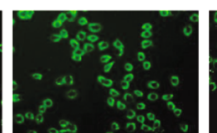

$\mathrm{OA}$

(c)

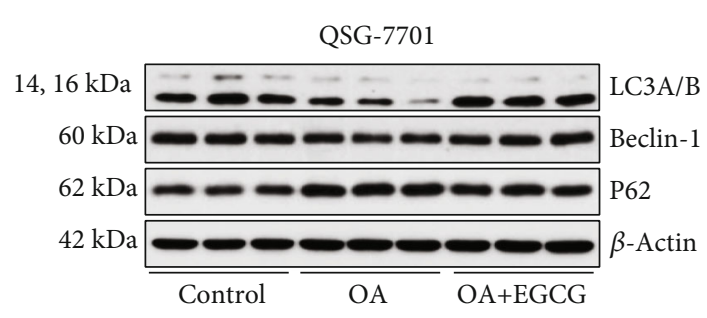

L02

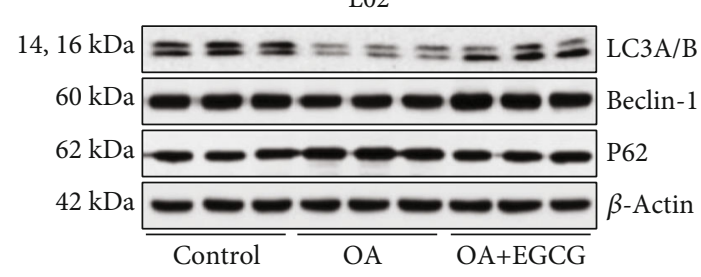

(e)
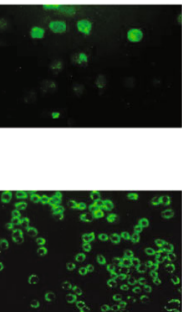

$\mathrm{OA}+\mathrm{EGCG}$

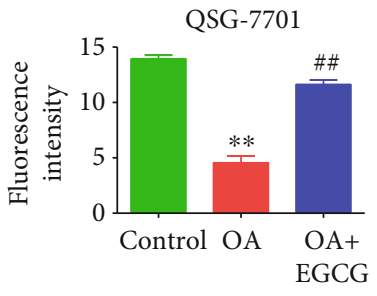

(d)
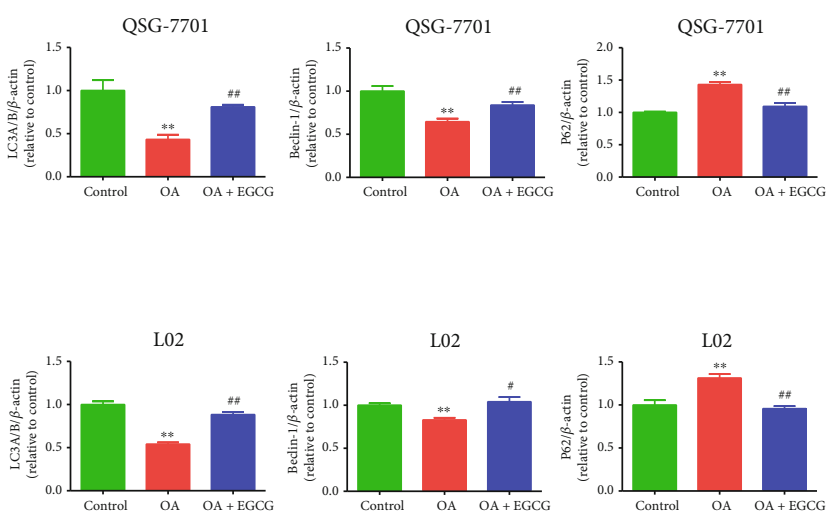

(f)

FIGURE 4: Effects of EGCG on the autophagy of OA-treated QSG-7701 and L02 cells. (a) GFP-RFP-LC3-transfected QSG-7701 and L02 cells were examined by fluorescence microscopy; original magnification $\times 1000$. (b) The ratios of red and yellow dots to transfected cells were calculated. (c) Representative photographs of MDC staining. (d) The fluorescence intensity was analyzed. (e) Western blot analysis for the expression levels of LC3A/B, beclin-1, and P62 in each group. $\beta$-Actin was used as the loading control. (f) The densitometry analysis of each factor was performed in each group, normalized to the corresponding $\beta$-actin level. Data are presented as mean \pm SEM of three independent experiments; ${ }^{* *} P<0.01$ compared with the control group; ${ }^{\#} P<0.05,{ }^{\# \#} P<0.01$ compared with the OA group. 


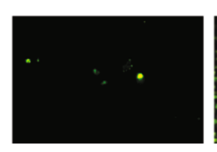

Control
QSG-7701

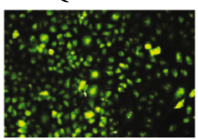

$\mathrm{OA}$

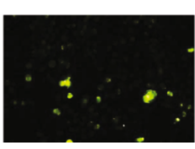

$\mathrm{OA}+\mathrm{EGCG}$

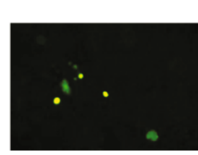

Control

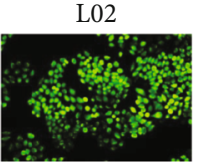

$\mathrm{OA}$

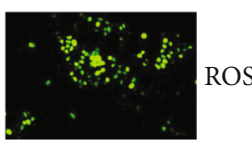

$\mathrm{OA}+\mathrm{EGCG}$

(a)
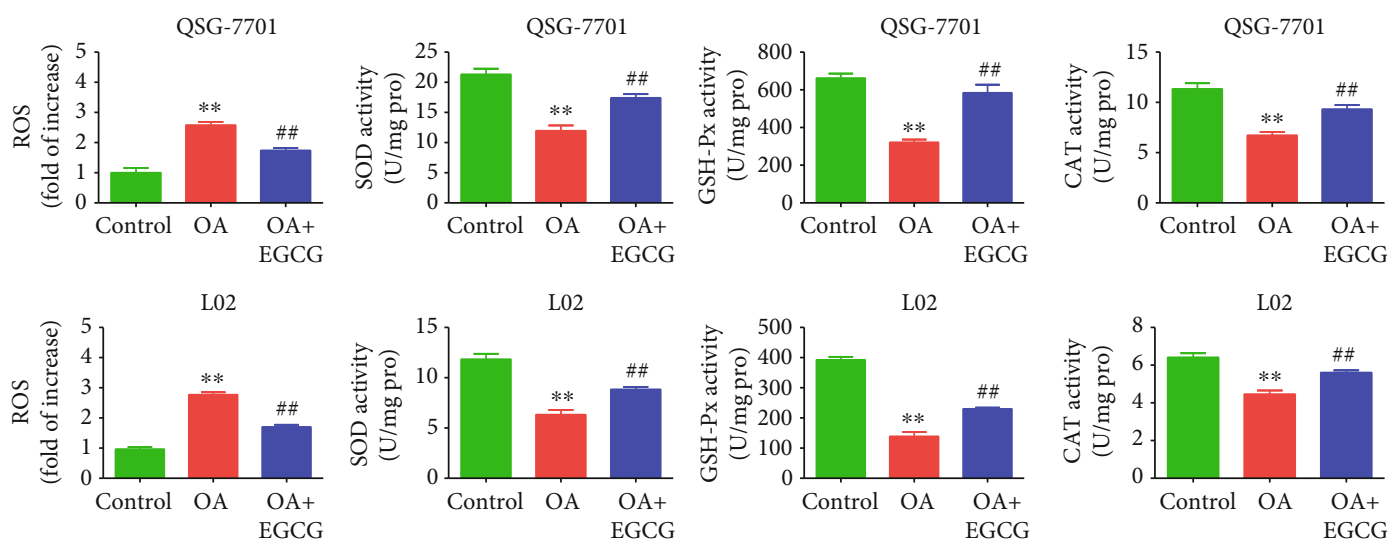

(b)
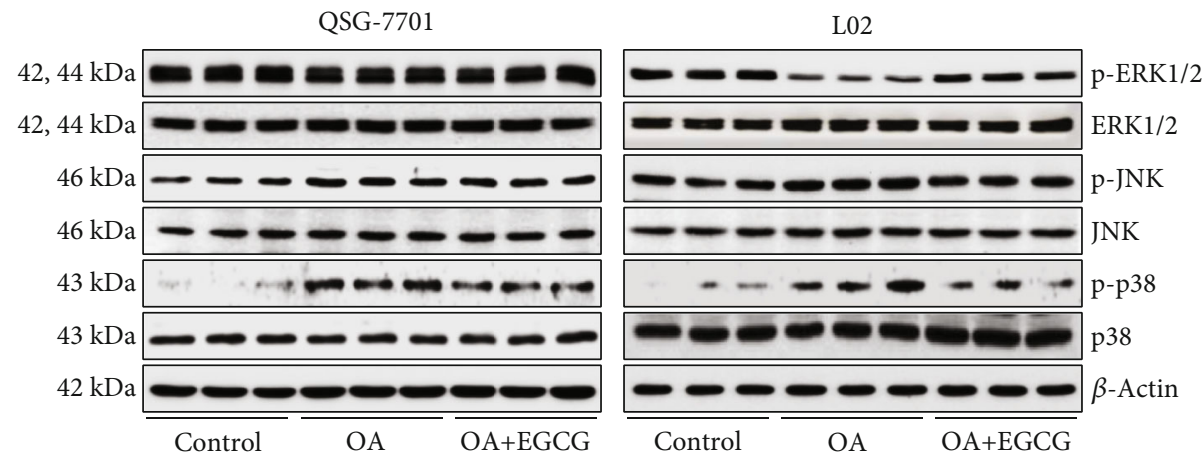

(c)
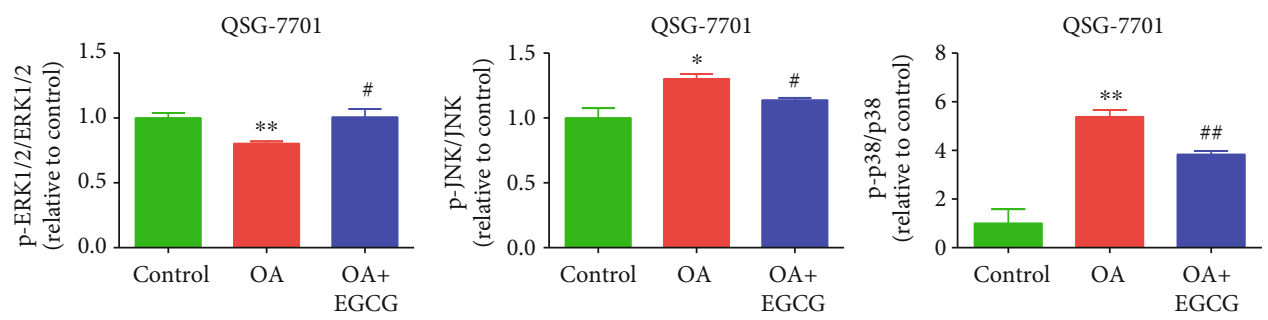

L02
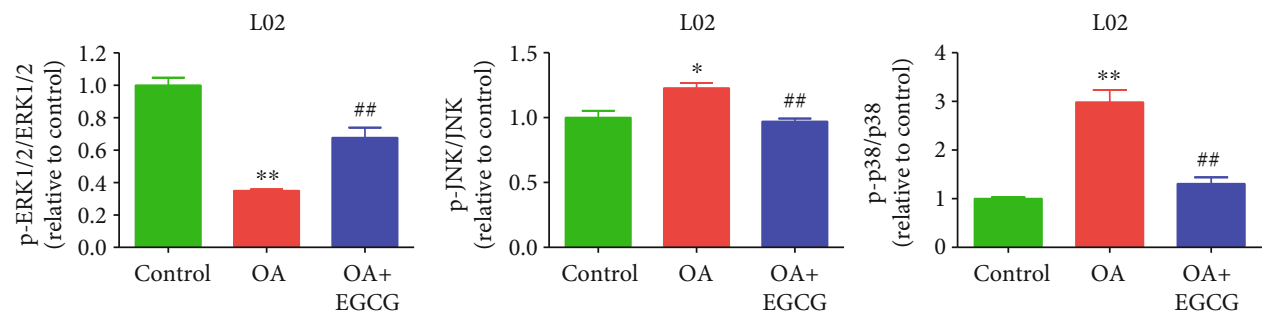

(d)

FIGURE 5: Effects of EGCG on the ROS/MAPK signaling pathway in OA-treated QSG-7701 and L02 cells. (a) The intracellular ROS production was detected using the fluorescent probe DCF-DA (shown in green; original magnification, $\times 400$ ). (b) The intracellular ROS production and the activities of SOD, GSH-Px, and CAT were measured. (c) The protein expressions of ERK1/2, p-ERK1/2, JNK, p-JNK, p38, and p-p38 were analyzed by Western blot. $\beta$-Actin was used as the loading control. (d) The densitometry analysis of each factor was performed in each group, normalized to the corresponding $\beta$-actin level. Data are presented as mean \pm SEM of three independent experiments; ${ }^{*} P<0.05,{ }^{* *} P<0.01$ compared with the control group; ${ }^{\#} P<0.05,{ }^{\# *} P<0.01$ compared with the OA group. 

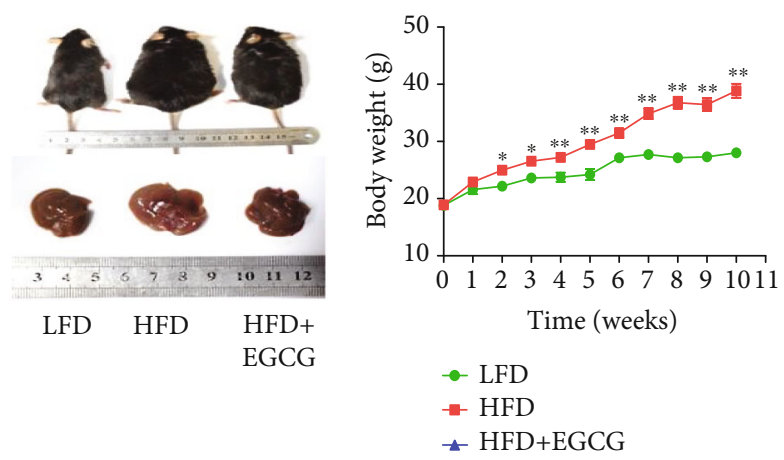

(a)

(b)
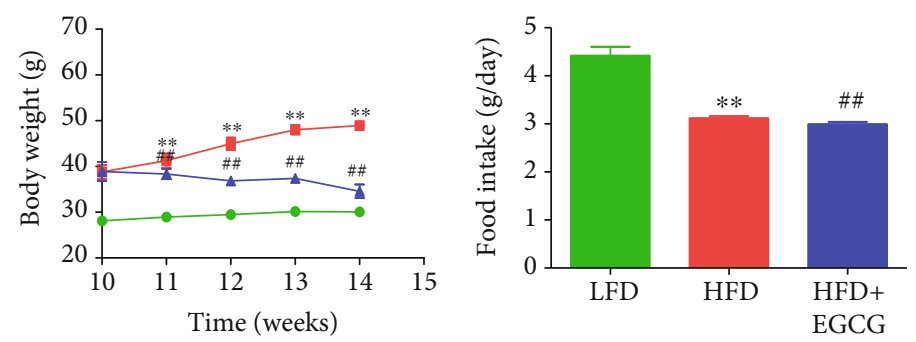

(c)

(d)
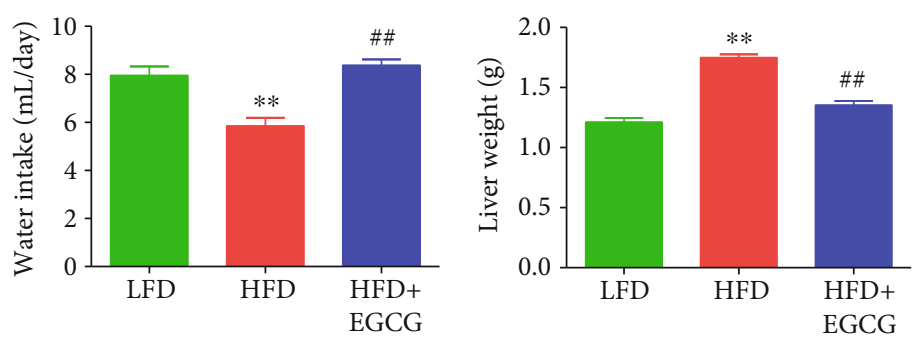

(e)

(f)
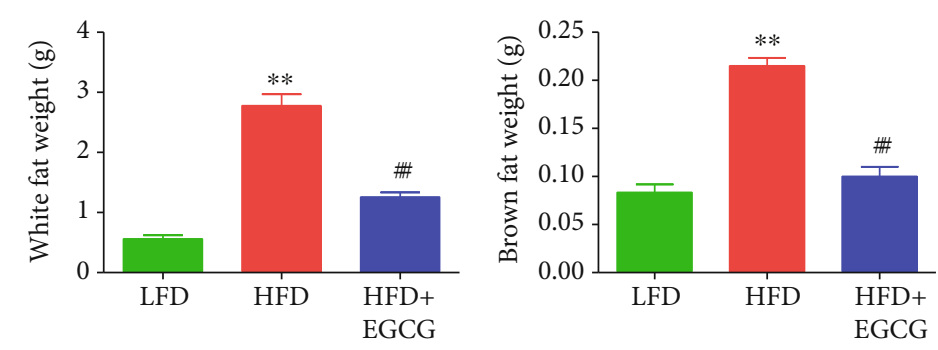

(g)

(h)
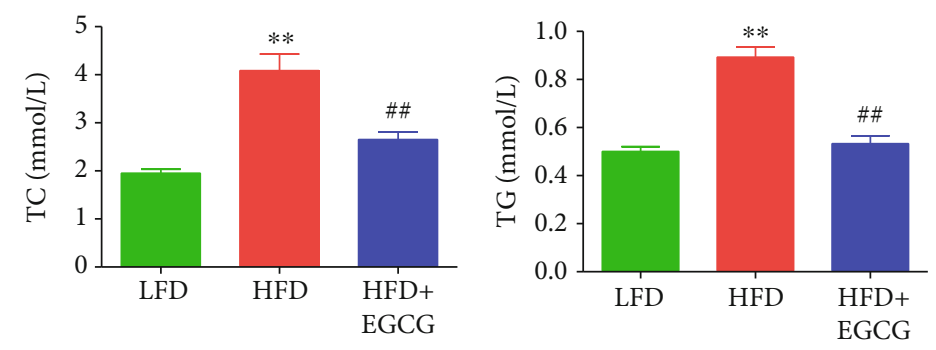

(i)

(j)

Figure 6: Continued. 


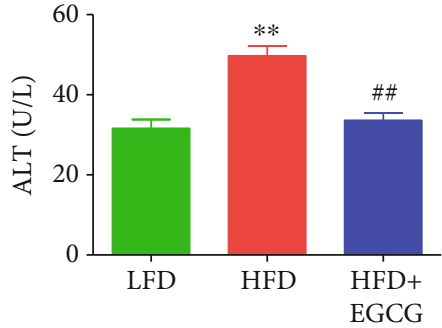

(k)

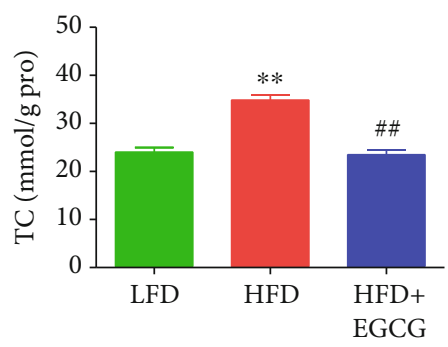

$(\mathrm{m})$

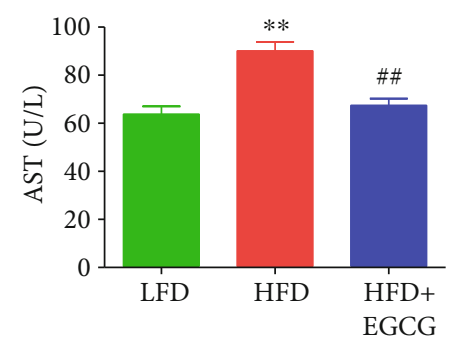

(1)

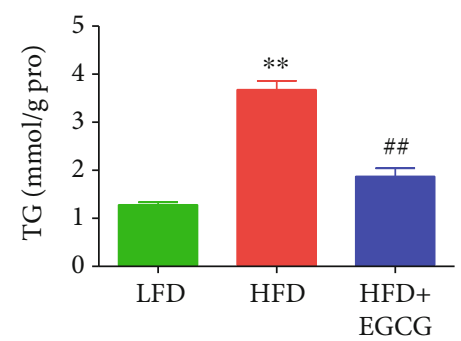

(n)

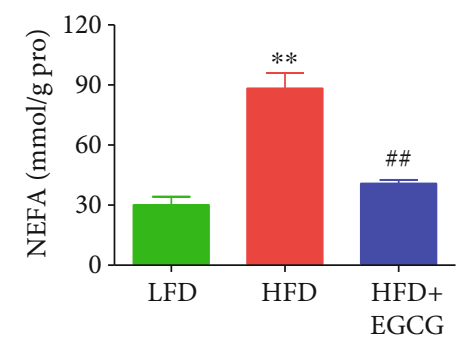

(o)

FIGURE 6: Effects of EGCG on HFD-induced NAFLD in mice. (a) Representative photographs of mice in each group. (b, c) The body weights of mice were measured. (d, e) Food intake and water intake were determined. (f-h) The liver weight, white fat weight, and brown fat weight were calculated. (i-l) The levels of TC, TG, ALT, and AST in the plasma of mice were detected. (m-o) The expression levels of TC, TG, and NEFA in the liver of mice were detected. Data are presented as mean $\pm \operatorname{SEM}(n=6) .{ }^{*} P<0.05,{ }^{* *} P<0.01$ compared with the control group; ${ }^{\# \#} P<0.01$ compared with the OA group.

stimulating autophagic flux in liver cells and the mouse liver [57]. Our data showed that EGCG could increase autophagy in OA-treated liver cells, indicating that autophagic activation can serve as a potential therapeutic target for NAFLD.

It has been shown that low concentrations of intracellular ROS are necessary for many physiological roles including signal transduction and cell proliferation. Nevertheless, ROS overproduction can induce oxidative stress and cellular redox imbalance, thus ultimately affecting many cellular functions $[41,58]$. Our data indicated that OA increased ROS levels and decreased the activities of GSH-Px, SOD, and CAT, which were consistent with the results of a previous study [59]. The effects were significantly reversed by the treatment with EGCG. In mammalian cells, three major types of MAP kinases are present: ERK, p38, and JNK, which are associated with EGCG interaction in the MAPK pathway $[60,61]$. MAPK cascades play key roles in the progression of NAFLD, and elevation of ROS activates the MAPK pathway [38, 41, $62]$. It has been revealed that intraperitoneal administration of EGCG $(5 \mathrm{mg} / \mathrm{kg})$ for 14 days inhibits phosphorylation of ERK, JNK, and p38 in animals with artificial unilateral ure- teral obstruction [63]. Another study indicates that MAPK and hypoxia-inducible factor- $1 \alpha$ are decreased after the treatment with EGCG, suggesting that EGCG could suppress MAPK-related oxidative stress [64]. A recent study has shown that EGCG can increase the expression levels of antioxidant enzymes, reverse the increase of ROS production, and regulate mitochondrial-involved autophagy [65]. Furthermore, EGCG could prevent $\alpha$ TC1- 6 cells from $\mathrm{H}_{2} \mathrm{O}_{2}$ induced ROS production via the activation of Akt signaling and suppression of the p38 and JNK pathway [66]. In this study, OA upregulated the expression levels of $\mathrm{p}$-JNK and p-p38 but downregulated the levels of p-ERK1/2. However, administration of EGCG remarkably reversed the levels of the proteins. Furthermore, the ROS/MAPK pathway is an important signaling cascade which can mediate the processes of apoptosis and autophagy in mammalian cells $[67,68]$. In sum, the data suggest that EGCG can reduce apoptosis and induce autophagy possibly through the ROS/MAPK pathway in OA-treated liver cells.

In this study, a mouse model of HFD-induced NAFLD was used to imitate unhealthy dietary habits. Our results 


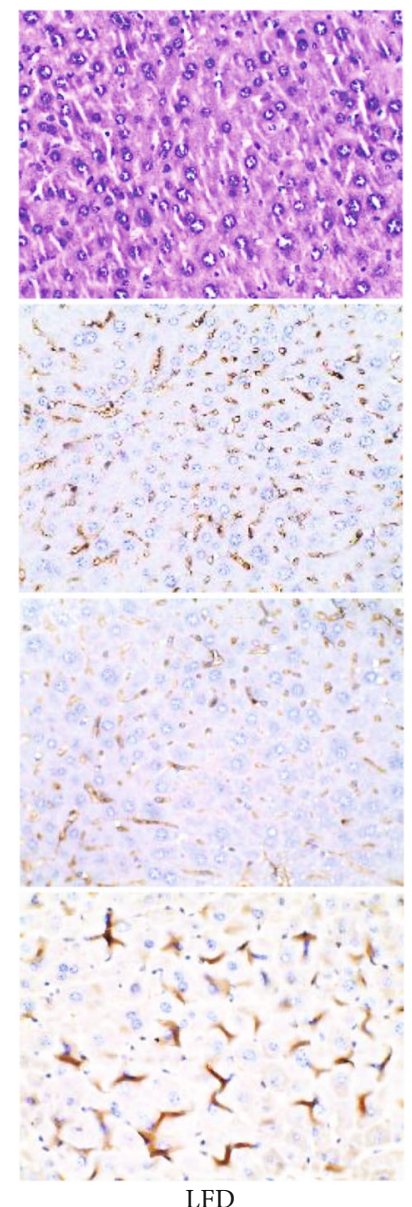

LFD

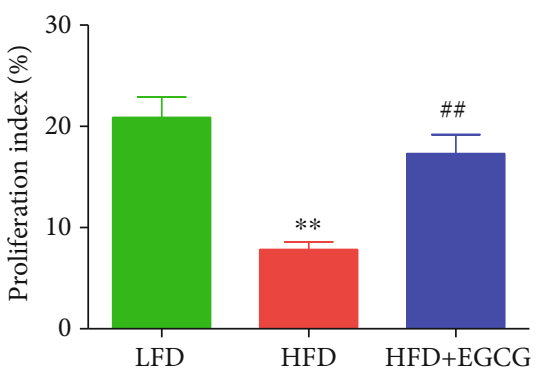

(b)
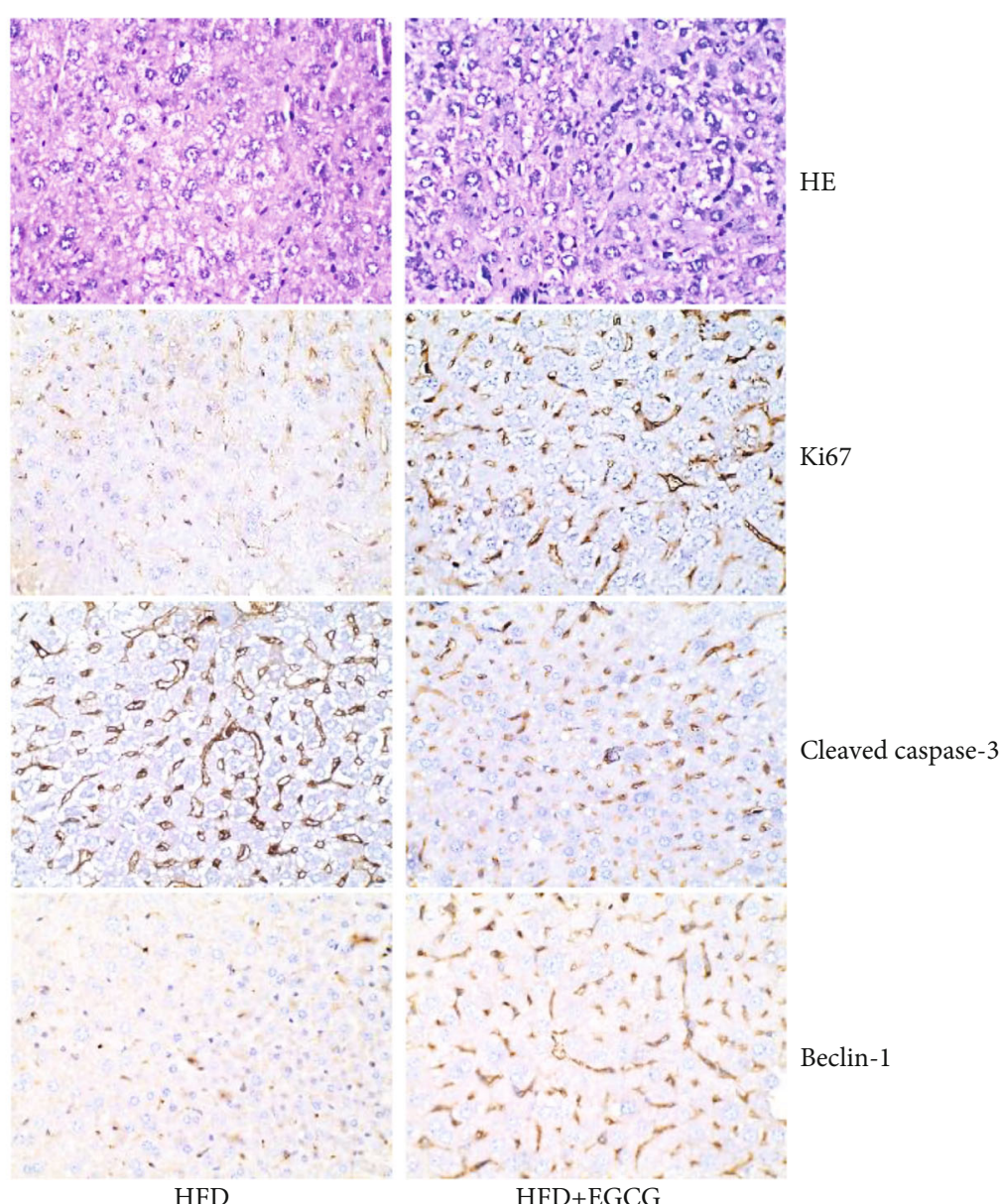

(a)

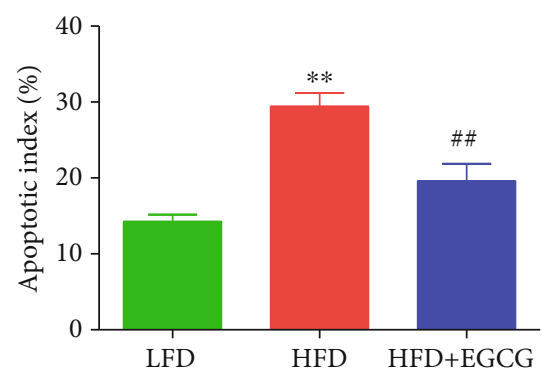

(c)

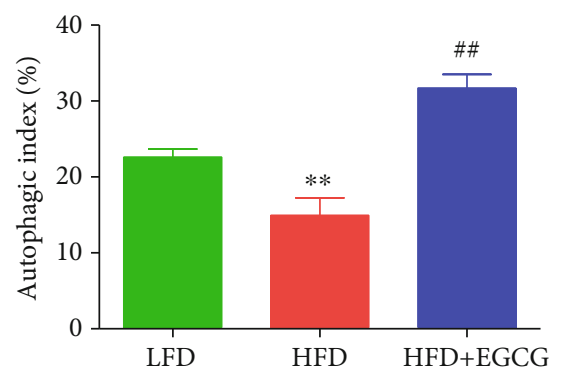

(d)

FIGURE 7: Effects of EGCG on the proliferation, apoptosis, and autophagy in liver tissues of the NAFLD mice. (a) Representative photographs of HE, Ki67, cleaved caspase-3, and beclin-1 staining in the liver of mice; original magnification $\times 400$. (b-d) The proliferation index, apoptotic index, and autophagic index were calculated. Data are presented as mean $\pm \operatorname{SEM}(n=3) .{ }^{* *} P<0.01$ compared with the control group; ${ }^{\# \#} P<0.01$ compared with the OA group.

suggested that HFD feeding induced obvious increases in body weight, liver weight, white fat weight, and brown fat weight, as well as the levels of TC, TG, ALT, and AST in the plasma of mice, indicating the successful establishment of the NAFLD model. It has been reported that NAFLD patients tend to have higher TC and TG levels [69]. EGCG markedly decreased the levels of TG and TC in both the plasma and the liver. Furthermore, ALT and AST are important indicators of liver damage in NAFLD [70]. Administra- tion of EGCG significantly alleviated liver damage in HFDfed mice by reducing ALT and AST levels. The flux of NEFA can be delivered to hepatocytes for TG synthesis, resulting in the development of NAFLD [71]. Treatment with EGCG decreased the level of NEFA in the liver of HFD-fed mice. Moreover, EGCG can increase the proliferation and autophagy but decrease the apoptosis in the liver of HFD-fed mice. The data together indicate that EGCG might alleviate HFD-induced NAFLD by inhibiting apoptosis and promoting autophagy. 
In summary, our data suggest that EGCG can alleviate HFD-induced NAFLD through inhibition of apoptosis and promotion of autophagy possibly via the ROS/MAPK pathway. EGCG could be developed as an effective agent for the treatment of NAFLD.

\section{Abbreviations}

\begin{tabular}{|c|c|}
\hline EGCG: & Epigallocatechin-3-gallate \\
\hline NAFLD: & Nonalcoholic fatty liver disease \\
\hline HFD: & High-fat diet \\
\hline OA: & Oleic acid \\
\hline DMEM: & Dulbecco's modified Eagle's medium \\
\hline FBS: & Fetal bovine serum \\
\hline BSA: & Bovine serum albumin \\
\hline ORO: & Oil red O \\
\hline EdU: & 5-Ethynyl-2' -deoxyuridine \\
\hline CCK-8: & Cell counting kit- 8 \\
\hline PI: & Propidium iodide \\
\hline GFP: & Green fluorescent protein \\
\hline RFP: & Red fluorescent protein \\
\hline MAP1LC3/LC3: & $\begin{array}{l}\text { Microtubule-associated protein } 1 \text { light } \\
\text { chain } 3\end{array}$ \\
\hline MDC: & Monodansylcadaverine \\
\hline ROS: & Reactive oxygen species \\
\hline SOD: & Superoxide dismutase \\
\hline CAT: & Catalase \\
\hline GSH-Px: & Glutathione peroxidase \\
\hline CDK: & Cyclin-dependent kinase \\
\hline ERK1/2: & $\begin{array}{l}\text { Extracellular signal-regulated protein } \\
\text { kinase } 1 / 2\end{array}$ \\
\hline p: & Phospho \\
\hline JNK: & Jun N-terminal kinase \\
\hline CST: & Cell Signaling Technology \\
\hline Bcl-2: & B-cell lymphoma-2 \\
\hline Bax: & Bcl-2-associated X protein \\
\hline Bcl-xl: & B-cell lymphoma-extra large \\
\hline Bad: & $\mathrm{Bcl}-\mathrm{xl} / \mathrm{Bcl}-2$-associated death promoter \\
\hline PARP: & Poly-ADP-ribose polymerase \\
\hline LFD: & Low-fat diet \\
\hline TG: & Triglyceride \\
\hline TC: & Total cholesterol \\
\hline ALT: & Alanine aminotransferase \\
\hline AST: & Aspartate aminotransferase \\
\hline NEFA: & Nonesterified fatty acid \\
\hline HE: & Hematoxylin and eosin \\
\hline IHC: & Immunohistochemistry \\
\hline MAPK: & Mitogen-activated protein kinase. \\
\hline
\end{tabular}

\section{Data Availability}

All data generated or analyzed in this study are included in this published article.

\section{Ethical Approval}

The animal experiment was approved by the Committee of Medical Ethics and Welfare for Experimental Animals of Henan University School of Medicine (HUSOM-2017-208).

\section{Conflicts of Interest}

The authors declare that there is no conflict of interests.

\section{Authors' Contributions}

D. W., Z. X., A. J., and Y. L. conceived and designed the experiments; Z. L., Y. W., Q. Z., J. L., and P. Z. performed the experiments and prepared the figures; and D. W. wrote the main manuscript text. All authors reviewed and approved the manuscript.

\section{Acknowledgments}

The study was supported by grants from the National Natural Science Foundation of China (Nos. 81802718, U1504817, and 81870591), the Foundation of Science \& Technology Department of Henan Province, China (Nos. 202102310480, 182102310335, and 192102310151), the Training Program for Young Backbone Teachers of Institutions of Higher Learning in Henan Province, China (No. 2020GGJS038), and the Science Foundation for Young Talents of Henan University College of Medicine, China (No. 2019013).

\section{References}

[1] D. Wang, Y. Wei, T. Wang et al., "Melatonin attenuates (-)-epigallocatehin-3-gallate-triggered hepatotoxicity without compromising its downregulation of hepatic gluconeogenic and lipogenic genes in mice," Journal of Pineal Research, vol. 59, no. 4, pp. 497-507, 2015.

[2] R. Y. Gan, H. B. Li, Z. Q. Sui, and H. Corke, "Absorption, metabolism, anti-cancer effect and molecular targets of epigallocatechin gallate (EGCG): an updated review," Critical Reviews in Food Science and Nutrition, vol. 58, no. 6, pp. 924-941, 2018.

[3] H. S. Kim, M. J. Quon, and J. A. Kim, "New insights into the mechanisms of polyphenols beyond antioxidant properties; lessons from the green tea polyphenol, epigallocatechin 3-gallate," Redox Biology, vol. 2, pp. 187-195, 2014.

[4] J. Steinmann, J. Buer, T. Pietschmann, and E. Steinmann, "Anti-infective properties of epigallocatechin-3-gallate (EGCG), a component of green tea," British Journal of Pharmacology, vol. 168, no. 5, pp. 1059-1073, 2013.

[5] K. Liang, J. E. Chung, S. J. Gao, N. Yongvongsoontorn, and M. Kurisawa, "Highly augmented drug loading and stability of micellar nanocomplexes composed of doxorubicin and poly(ethylene glycol)-green tea catechin conjugate for cancer therapy," Advanced Materials, vol. 30, no. 14, article e1706963, 2018.

[6] K. Liang, K. H. Bae, F. Lee et al., "Self-assembled ternary complexes stabilized with hyaluronic acid-green tea catechin conjugates for targeted gene delivery," Journal of Controlled Release, vol. 226, pp. 205-216, 2016.

[7] S. J. Hyung, A. S. DeToma, J. R. Brender et al., "Insights into antiamyloidogenic properties of the green tea extract (-)-epigallocatechin-3-gallate toward metal-associated amyloid- $\beta$ species," Proceedings of the National Academy of Sciences, vol. 110, no. 10, pp. 3743-3748, 2013.

[8] S. Chikara, L. D. Nagaprashantha, J. Singhal, D. Horne, S. Awasthi, and S. S. Singhal, "Oxidative stress and dietary 
phytochemicals: role in cancer chemoprevention and treatment," Cancer Letters, vol. 413, pp. 122-134, 2018.

[9] R. Kanlaya and V. Thongboonkerd, "Protective effects of epigallocatechin-3-gallate from green tea in various kidney diseases," Advances in Nutrition, vol. 10, no. 1, pp. 112-121, 2019.

[10] J. E. Chung, S. Tan, S. J. Gao et al., "Self-assembled micellar nanocomplexes comprising green tea catechin derivatives and protein drugs for cancer therapy," Nature Nanotechnology, vol. 9, no. 11, pp. 907-912, 2014.

[11] L. G. Xiong, Y. J. Chen, J. W. Tong, Y. S. Gong, J. A. Huang, and Z. H. Liu, "Epigallocatechin-3-gallate promotes healthy lifespan through mitohormesis during early-to-mid adulthood in Caenorhabditis elegans," Redox Biology, vol. 14, pp. 305315, 2018.

[12] Z. S. Liu, H. Cai, W. Xue et al., "G3BP1 promotes DNA binding and activation of cGAS," Nature Immunology, vol. 20, no. 1, pp. 18-28, 2019.

[13] N. Calland, A. Albecka, S. Belouzard et al., "(-)-Epigallocatechin-3-gallate is a new inhibitor of hepatitis C virus entry," Hepatology, vol. 55, no. 3, pp. 720-729, 2012.

[14] G. Targher and C. D. Byrne, "Non-alcoholic fatty liver disease: an emerging driving force in chronic kidney disease," Nature Reviews. Nephrology, vol. 13, no. 5, pp. 297-310, 2017.

[15] E. Vilar-Gomez, L. Calzadilla-Bertot, V. Wai-Sun Wong et al., "Fibrosis severity as a determinant of cause-specific mortality in patients with advanced nonalcoholic fatty liver disease: a multi-national cohort study," Gastroenterology, vol. 155, no. 2, pp. 443-457.e17, 2018.

[16] Z. M. Younossi, "Non-alcoholic fatty liver disease - a global public health perspective," Journal of Hepatology, vol. 70, no. 3, pp. 531-544, 2019.

[17] N. Kuzu, I. H. Bahcecioglu, A. F. Dagli, I. H. Ozercan, B. Ustündag, and K. Sahin, "Epigallocatechin gallate attenuates experimental non-alcoholic steatohepatitis induced by high fat diet," Journal of Gastroenterology and Hepatology, vol. 23, no. 8, pp. e465-e470, 2008.

[18] L. Gan, Z. J. Meng, R. B. Xiong et al., "Green tea polyphenol epigallocatechin-3-gallate ameliorates insulin resistance in non-alcoholic fatty liver disease mice," Acta Pharmacologica Sinica, vol. 36, no. 5, pp. 597-605, 2015.

[19] Y. Ding, X. Sun, Y. Chen, Y. Deng, and K. Qian, “Epigallocatechin gallate attenuated non-alcoholic steatohepatitis induced by methionine- and choline-deficient diet," European Journal of Pharmacology, vol. 761, pp. 405-412, 2015.

[20] S. Vidyashankar, R. Sandeep Varma, and P. S. Patki, “Quercetin ameliorate insulin resistance and up-regulates cellular antioxidants during oleic acid induced hepatic steatosis in HepG2 cells," Toxicology In Vitro, vol. 27, no. 2, pp. 945-953, 2013.

[21] T. Qiu, P. Pei, X. Yao et al., "Taurine attenuates arsenicinduced pyroptosis and nonalcoholic steatohepatitis by inhibiting the autophagic-inflammasomal pathway," Cell Death \& Disease, vol. 9, no. 10, p. 946, 2018.

[22] D. Wu, J. Li, Q. Zhang et al., "Exogenous hydrogen sulfide regulates the growth of human thyroid carcinoma cells," Oxidative Medicine and Cellular Longevity, vol. 2019, no. 6927298, 18 pages, 2019.

[23] Y. Zhai, P. Lin, Z. Feng et al., "TNFAIP3-DEPTOR complex regulates inflammasome secretion through autophagy in ankylosing spondylitis monocytes," Autophagy, vol. 14, no. 9, pp. 1629-1643, 2018.
[24] Y. Wang, H. Nie, X. Zhao, Y. Qin, and X. Gong, "Bicyclol induces cell cycle arrest and autophagy in HepG2 human hepatocellular carcinoma cells through the PI3K/AKT and Ras/Raf/MEK/ERK pathways," BMC Cancer, vol. 16, no. 1, p. 742, 2016.

[25] Y. Yuan, D. Ding, N. Zhang et al., “TNF- $\alpha$ induces autophagy through ERK1/2 pathway to regulate apoptosis in neonatal necrotizing enterocolitis model cells IEC-6," Cell Cycle, vol. 17, no. 11, pp. 1390-1402, 2018.

[26] Y. Xie, S. Li, L. Sun et al., "Fungal immunomodulatory protein from Nectria haematococca suppresses growth of human lung adenocarcinoma by inhibiting the PI3K/Akt pathway," International Journal of Molecular Sciences, vol. 19, no. 11, p. 3429, 2018.

[27] Q. Shen, J. W. Eun, K. Lee et al., "Barrier to autointegration factor 1, procollagen-lysine, 2-oxoglutarate 5-dioxygenase 3, and splicing factor $3 \mathrm{~b}$ subunit 4 as early-stage cancer decision markers and drivers of hepatocellular carcinoma," Hepatology, vol. 67, no. 4, pp. 1360-1377, 2018.

[28] P. Pitchakarn, S. Suzuki, K. Ogawa et al., "Induction of G1 arrest and apoptosis in androgen-dependent human prostate cancer by Kuguacin J, a triterpenoid from Momordica charantia leaf," Cancer Letters, vol. 306, no. 2, pp. 142-150, 2011.

[29] M. Chen, X. Wang, D. Zha et al., “Apigenin potentiates TRAIL therapy of non-small cell lung cancer_via_ upregulating DR4/DR5 expression in a p53-dependent manner," Scientific Reports, vol. 6, no. 1, article 35468, 2016.

[30] S. M. Man and T. D. Kanneganti, "Converging roles of caspases in inflammasome activation, cell death and innate immunity," Nature Reviews. Immunology, vol. 16, no. 1, pp. 7-21, 2016.

[31] D. Wu, W. Tian, J. Li et al., "Peptide P11 suppresses the growth of human thyroid carcinoma by inhibiting the PI3K/AKT/mTOR signaling pathway," Molecular Biology Reports, vol. 46, no. 3, pp. 2665-2678, 2019.

[32] J. Li, R. Zhu, K. Chen et al., "Potent and specific Atg8-targeting autophagy inhibitory peptides from giant ankyrins," Nature Chemical Biology, vol. 14, no. 8, pp. 778-787, 2018.

[33] L. Galluzzi, E. H. Baehrecke, A. Ballabio et al., "Molecular definitions of autophagy and related processes," The EMBO Journal, vol. 36, no. 13, pp. 1811-1836, 2017.

[34] S. Lépine, J. C. Allegood, M. Park, P. Dent, S. Milstien, and S. Spiegel, "Sphingosine-1-phosphate phosphohydrolase-1 regulates ER stress-induced autophagy," Cell Death and Differentiation, vol. 18, no. 2, pp. 350-361, 2011.

[35] D. J. Klionsky, K. Abdelmohsen, A. Abe et al., "Guidelines for the use and interpretation of assays for monitoring autophagy (3rd edition)," Autophagy, vol. 12, no. 1, pp. 1-222, 2016.

[36] D. Y. Wang, Y. Hong, Y. G. Chen et al., "PEST-containing nuclear protein regulates cell proliferation, migration, and invasion in lung adenocarcinoma," Oncogene, vol. 8, no. 3, p. 22, 2019.

[37] B. Nuvoli, E. Camera, A. Mastrofrancesco, S. Briganti, and R. Galati, "Modulation of reactive oxygen species via ERK and STAT3 dependent signalling are involved in the response of mesothelioma cells to exemestane," Free Radical Biology \& Medicine, vol. 115, pp. 266-277, 2018.

[38] G. Y. Zhang, D. Lu, S. F. Duan et al., "Hydrogen sulfide alleviates lipopolysaccharide-induced diaphragm dysfunction in rats by reducing apoptosis and inflammation through ROS/MAPK and TLR4/NF- $\kappa \mathrm{B}$ signaling pathways," Oxidative 
Medicine and Cellular Longevity, vol. 2018, no. 9647809, 15 pages, 2018.

[39] C. Y. Li, H. S. Hao, Y. H. Zhao et al., "Melatonin improves the fertilization capacity of sex-sorted bull sperm by inhibiting apoptosis and increasing fertilization capacitation via MT1," International Journal of Molecular Sciences, vol. 20, no. 16, p. 3921, 2019.

[40] Z. Zhang, Z. Ren, S. Chen et al., "ROS generation and JNK activation contribute to 4-methoxy-TEMPO-induced cytotoxicity, autophagy, and DNA damage in HepG2 cells," Archives of Toxicology, vol. 92, no. 2, pp. 717-728, 2018.

[41] D. Wu, N. Luo, L. Wang et al., "Hydrogen sulfide ameliorates chronic renal failure in rats by inhibiting apoptosis and inflammation through ROS/MAPK and NF- $\kappa$ B signaling pathways," Scientific Reports, vol. 7, no. 1, p. 455, 2017.

[42] Z. Liu, J. Wang, C. Guo, and X. Fan, "MicroRNA-21 mediates epithelial-mesenchymal transition of human hepatocytes via PTEN/Akt pathway," Biomedicine \& Pharmacotherapy, vol. 69 , pp. 24-28, 2015.

[43] D. Wu, M. Li, W. Tian et al., "Hydrogen sulfide acts as a double-edged sword in human hepatocellular carcinoma cells through EGFR/ERK/MMP-2 and PTEN/AKT signaling pathways," Scientific Reports, vol. 7, no. 1, p. 5134, 2017.

[44] Q. Chu, S. Zhang, M. Chen et al., "Cherry anthocyanins regulate NAFLD by promoting autophagy pathway," Oxidative Medicine and Cellular Longevity, vol. 2019, no. 4825949, 16 pages, 2019.

[45] Y. Liu, Y. Cheng, J. Li, Y. Wang, and Y. Liu, "Epoxy stearic acid, an oxidative product derived from oleic acid, induces cytotoxicity, oxidative stress, and apoptosis in HepG2 cells," Journal of Agricultural and Food Chemistry, vol. 66, no. 20, pp. 5237-5246, 2018.

[46] R. Singh, A. Letai, and K. Sarosiek, "Regulation of apoptosis in health and disease: the balancing act of BCL-2 family proteins," Nature Reviews. Molecular Cell Biology, vol. 20, no. 3, pp. 175-193, 2019.

[47] D. Wu, W. Si, M. Wang, S. Lv, A. Ji, and Y. Li, "Hydrogen sulfide in cancer: friend or foe?," Nitric Oxide, vol. 50, pp. 38-45, 2015.

[48] D. Wu, Y. Gao, Y. Qi, L. Chen, Y. Ma, and Y. Li, "Peptidebased cancer therapy: opportunity and challenge," Cancer Letters, vol. 351, no. 1, pp. 13-22, 2014.

[49] Q. Dong, B. Yang, J. G. Han et al., “A novel hydrogen sulfidereleasing donor, HA-ADT, suppresses the growth of human breast cancer cells through inhibiting the PI3K/AKT/mTOR and Ras/Raf/MEK/ERK signaling pathways," Cancer Letters, vol. 455, pp. 60-72, 2019.

[50] L. Pang, K. Liu, D. Liu et al., "Differential effects of reticulophagy and mitophagy on nonalcoholic fatty liver disease," Cell Death \& Disease, vol. 9, no. 2, p. 90, 2018.

[51] T. P. Patel, K. Rawal, S. Soni, and S. Gupta, "Swertiamarin ameliorates oleic acid induced lipid accumulation and oxidative stress by attenuating gluconeogenesis and lipogenesis in hepatic steatosis," Biomedicine \& Pharmacotherapy, vol. 83, pp. 785-791, 2016.

[52] D. Wu, H. Wang, T. Teng, S. Duan, A. Ji, and Y. Li, "Hydrogen sulfide and autophagy: a double edged sword," Pharmacological Research, vol. 131, pp. 120-127, 2018.

[53] A. J. Clarke and A. K. Simon, "Autophagy in the renewal, differentiation and homeostasis of immune cells," Nature Reviews. Immunology, vol. 19, no. 3, pp. 170-183, 2019.
[54] A. González-Rodríguez, R. Mayoral, N. Agra et al., "Impaired autophagic flux is associated with increased endoplasmic reticulum stress during the development of NAFLD," Cell Death of Disease, vol. 5, no. 4, article e1179, 2014.

[55] L. Zhang, Z. Yao, and G. Ji, "Herbal extracts and natural products in alleviating non-alcoholic fatty liver disease via activating autophagy," Frontiers in Pharmacology, vol. 9, p. 1459, 2018.

[56] T. Ueno and M. Komatsu, "Autophagy in the liver: functions in health and disease," Nature Reviews. Gastroenterology \& Hepatology, vol. 14, no. 3, pp. 170-184, 2017.

[57] J. Zhou, B. L. Farah, R. A. Sinha et al., "Epigallocatechin-3-gallate (EGCG), a green tea polyphenol, stimulates hepatic autophagy and lipid clearance," PLoS One, vol. 9, no. 1, article e87161, 2014.

[58] H. Cui, S. Wu, Y. Shang et al., "Pleurotus nebrodensis polysaccharide (PN50G) evokes A549 cell apoptosis by the ROS/AMPK/PI3K/AKT/mTOR pathway to suppress tumor growth," Food \& Function, vol. 7, no. 3, pp. 1616-1627, 2016.

[59] H. Su, Y. Li, D. Hu et al., "Procyanidin B2 ameliorates free fatty acids-induced hepatic steatosis through regulating TFEBmediated lysosomal pathway and redox state," Free Radical Biology \& Medicine, vol. 126, pp. 269-286, 2018.

[60] J. S. Arthur and S. C. Ley, "Mitogen-activated protein kinases in innate immunity," Nature Reviews Immunology, vol. 13, no. 9, pp. 679-692, 2013.

[61] M. Sharifi-Rad, R. Pezzani, M. Redaelli et al., "Preclinical pharmacological activities of epigallocatechin-3-gallate in signaling pathways: an update on cancer," Molecules, vol. 25, no. 3, p. $467,2020$.

[62] X. Shen, H. Guo, J. Xu, and J. Wang, "Inhibition of lncRNA HULC improves hepatic fibrosis and hepatocyte apoptosis by inhibiting the MAPK signaling pathway in rats with nonalcoholic fatty liver disease," Journal of Cellular Physiology, vol. 234, no. 10, pp. 18169-18179, 2019.

[63] Y. Wang, N. Liu, X. Bian et al., "Epigallocatechin-3-gallate reduces tubular cell apoptosis in mice with ureteral obstruction," The Journal of Surgical Research, vol. 197, no. 1, pp. 145-154, 2015.

[64] J. Chen, J. Xu, J. Li et al., "Epigallocatechin-3-gallate attenuates lipopolysaccharide-induced mastitis in rats via suppressing MAPK mediated inflammatory responses and oxidative stress," International Immunopharmacology, vol. 26, no. 1, pp. 147-152, 2015.

[65] E. Casanova, J. Salvadó, A. Crescenti, and A. Gibert-Ramos, "Epigallocatechin gallate modulates muscle homeostasis in type 2 diabetes and obesity by targeting energetic and redox pathways: a narrative review," International Journal of Molecular Sciences, vol. 20, no. 3, p. 532, 2019.

[66] T. Cao, X. Zhang, D. Yang et al., "Antioxidant effects of epigallocatechin-3-gallate on the aTC1-6 pancreatic alpha cell line," Biochemical and Biophysical Research Communications, vol. 495, no. 1, pp. 693-699, 2018.

[67] K. Wang, B. Chen, T. Yin et al., "N-Methylparoxetine blocked autophagic flux and induced apoptosis by activating ROSMAPK pathway in non-small cell lung cancer cells," International Journal of Molecular Sciences, vol. 20, no. 14, p. 3415, 2019.

[68] Y. Liu and D. Fan, "Ginsenoside Rg5 induces G2/M phase arrest, apoptosis and autophagy via regulating ROS-mediated MAPK pathways against human gastric cancer," Biochemical Pharmacology, vol. 168, pp. 285-304, 2019. 
[69] X. Zheng, L. Gong, R. Luo et al., "Serum uric acid and nonalcoholic fatty liver disease in non-obesity Chinese adults," Lipids in Health and Disease, vol. 16, no. 1, p. 202, 2017.

[70] M. Lazo, J. Rubin, J. M. Clark et al., “The association of liver enzymes with biomarkers of subclinical myocardial damage and structural heart disease," Journal of Hepatology, vol. 62, no. 4, pp. 841-847, 2015.

[71] H. S. Jeong, K. H. Kim, I. S. Lee et al., "Ginkgolide A ameliorates non-alcoholic fatty liver diseases on high fat diet mice," Biomedicine \& Pharmacotherapy, vol. 88, pp. 625-634, 2017. 